\title{
Molecular Machines for Quantum Error Correction
}

\author{
Thiago Guerreiro* \\ Department of Physics, Pontificia Universidade Católica, Rio de Janeiro, Rio de Janeiro 22451-900, Brazil
}

(Received 11 March 2021; accepted 6 August 2021; published 30 August 2021)

\begin{abstract}
Inspired by biological molecular machines, we explore the idea of an active quantum robot the purpose of which is to delay decoherence. A conceptual model capable of partially protecting arbitrary logical qubit states against single-physical-qubit errors is presented. Implementation of an instance of that model - the entanglement qubot - is proposed using laser-dressed Rydberg atoms. The dynamics of the system are studied using stochastic wave-function methods.
\end{abstract}

DOI: 10.1103/PRXQuantum.2.030336

\section{INTRODUCTION}

The living cell can be seen as a Brownian computer [1]. At its core, machines of molecular dimensions store, correct, and process information in the presence of noise, with the goal of keeping the state of the living creature away from thermodynamical equilibrium. The machinery of life [2] is responsible for gene expression, matter transport across the cell, and energy harvesting, among a vast number of other tasks [3]. An example of such molecular devices is RNA polymerase (RNAP): an enzyme with approximately 40000 atoms, roughly $10 \mathrm{~nm}$ of linear size, capable of synthesizing a strand of Ribonucleic acid (RNA) from a DNA template in the presence of Brownian noise, at error rates as low as $10^{-7}$ [4]. Molecular devices such as RNAP have inspired nanotechnology $[5,6]$ and various artificial molecular machines have been built, such as molecular ratchets [7], pumps [8], motors [9], and gene-editing tools [10].

A detailed unified understanding of biological molecular machines according to the tradition of theoretical physics is yet to be achieved [11] but there is little doubt that experimental [12] and computational methods [13] in physics play a key role in that endeavor. It is also expected that the coming age of quantum information processing will illuminate biological systems through the simulation of quantum chemistry [14] and quantum enhanced learning $[15,16]$. Conversely [17], we could ask whether biological molecular machines will inspire new ideas for engineering autonomous molecular-sized quantum information

\footnotetext{
*barbosa@puc-rio.br

Published by the American Physical Society under the terms of the Creative Commons Attribution 4.0 International license. Further distribution of this work must maintain attribution to the author(s) and the published article's title, journal citation, and DOI.
}

processing devices with the goal of keeping quantum states away from thermodynamical equilibrium. It is the purpose of this work to explore this idea.

A quantum molecular machine would be a device composed of at most a few thousand atoms, capable of autonomously storing, protecting, and/or processing quantum states in the presence of external decoherence and thermalization. We refer to these bioinspired devices as quantum robots, or qubots [18]. The devising of qubots is a problem in coherent quantum chemistry $[19,20]$, in much the same way that engineering artificial molecular machines is a problem in synthetic chemistry [21]. Hence, the ultracold atom [22] and the molecular toolbox [23,24] are expected to play key roles in the conception of these active quantum devices. As we shall see, qubots exploit open system dynamics to achieve their purpose and thus have a close connection to the idea of engineered environments constructed to produce desired quantum states [25-32]. Their nature, however, is much closer to that of artificial molecular ratchets and pumps that respond to the environment and consume resources to maintain nonequilibrium states [33].

In what follows, we explore various aspects around the idea of qubots. We begin by introducing a conceptual model for a quantum robot capable of partially protecting a logical qubit state against single-physicalqubit errors. It is interesting that the model can handle almost all combinations of phase and bit-flip errors since, as pointed out by Kitaev, it is generally easy to get rid of one kind of errors, but not both [34]. The construction is somewhat inspired by the surface code [35], except that here syndrome detection and correction are part of the dynamics of the system rather than a consequence of measurement followed by external conditional action. Next, a specific physical implementation of instances of the model based on laser-dressed Rydberg atoms is discussed. More specifically, we exhibit potential 
(a)

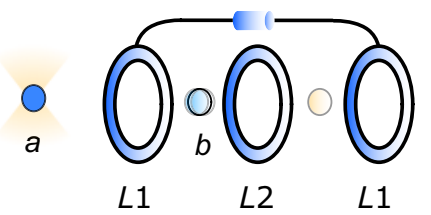

(b)

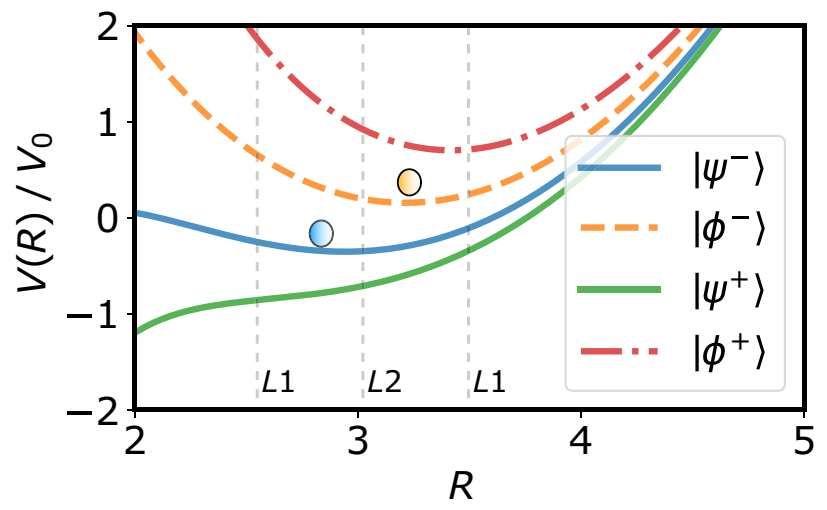

FIG. 1. (a) The schematics for a conceptual qubot model capable of partially protecting an arbitrary logical qubit state against decoherence. (b) An example of a possible potential landscape describing the interaction between the nucleus atoms; for this plot, the radial dependence of Eq. (2) is considered: $J_{\alpha}(R)=$ $\left(d^{2} / R^{3}\right) j_{\alpha}$, with $j_{y}=-3 j_{x}, j_{z}=6 j_{x}$.

landscapes implementing an entanglement qubot, a device that stabilizes a Bell state against single-qubit errors. The stabilized Bell state is only one possible state of the logical qubit but in this case we can view the qubot as preserving a maximally entangled state. An ensemble of entanglement qubots could therefore preserve vast amounts of entanglement, a useful resource. Simulation of the entanglement qubot dynamics is performed with the help of stochastic wave-function methods and we evaluate the effects of coupling the motional degrees of freedom of the robot to an external heat bath. We conclude with a discussion on potential future developments regarding active quantum matter.

\section{CONCEPTUAL MODEL}

We introduce the conceptual model of a quantum robot capable of protecting an arbitrary logical qubit state against errors. Our quantum robot consists of two parts, called the nucleus and the correctors [for a schematic representation, see Fig. 1(a)]. A pair of particles denoted $a$ and $b$ constitute the nucleus. Quantum information is stored in the internal spin degrees of freedom of the particles, taken to be two spin-1/2 systems with Hilbert space $\mathbb{C}^{2} \otimes \mathbb{C}^{2}$ and basis states denoted by $\{|0\rangle|0\rangle,|0\rangle|1\rangle,|1\rangle|0\rangle,|1\rangle|1\rangle\}$.

Particle $a$ is held fixed at the origin by an optical tweezer, while $b$ is subject to the potential

$$
V(R)=V_{t}(R)+V_{I}(R),
$$

where $R$ is the relative distance between $a$ and $b, V_{t}(R)$ is a trap potential for particle $b$, and

$$
V_{I}(R)=J_{z} Z_{a} Z_{b}+J_{x} X_{a} X_{b}+J_{y} Y_{a} Y_{b}
$$

is the interaction energy between the particles, where $X_{\lambda}$, $Y_{\lambda}$, and $Z_{\lambda}$ are the Pauli operators for particle $\lambda(=a, b)$ and the coefficients $J_{\alpha}=J_{\alpha}(R)$ form a spatial-dependent spin-spin interaction pattern. We assume for simplicity that particle $b$ can only move along the direction $\hat{R}$.

As an example of a trap potential, we may consider an optical tweezer

$$
V_{t}(R)=V_{0}(R-\delta)^{2},
$$

where $V_{0}$ and $\delta$ are constants. Tunneling outside the confining potential is considered negligible. Note also that dipole-dipole interactions among atoms and polar molecules is of the form given in Eq. (2) and that typically, for molecules [36,37] and spin impurities in diamond [38],

$$
J_{\alpha}=\left(d^{2} / R^{3}\right) j_{\alpha},
$$

where $d$ is the dipole moment [39] and $j_{\alpha}$ a proportionality constant, with $\alpha=x, y, z$. Through the remainder of this section, we consider this radial dependence as an illustration of the qubot functioning. Note, however, that effective spin interactions of the so-called $X Y Z$ form with more general radial dependencies can be engineered within a number of different systems, including trapped ions [40,41], atoms in dressed Rydberg states [42,43], and microwaveexcited polar molecules in optical lattices [44,45]. In Sec. III, an implementation using laser-dressed Rydberg atoms is discussed.

Bell states of the spins of the particles are eigenstates of $V_{I}$, with eigenvalues given by

$$
\begin{aligned}
& V_{I}\left|\psi^{-}\right\rangle=\left(-J_{x}-J_{y}-J_{z}\right)\left|\psi^{-}\right\rangle, \\
& V_{I}\left|\phi^{-}\right\rangle=\left(-J_{x}+J_{y}+J_{z}\right)\left|\phi^{-}\right\rangle, \\
& V_{I}\left|\psi^{+}\right\rangle=\left(J_{x}+J_{y}-J_{z}\right)\left|\psi^{+}\right\rangle, \\
& V_{I}\left|\phi^{+}\right\rangle=\left(J_{x}-J_{y}+J_{z}\right)\left|\phi^{+}\right\rangle .
\end{aligned}
$$

This implies that the total potential $V(R)$ exhibits collective spin-dependent landscapes.

As an example, consider the trap potential given in Eq. (3) and the spin pattern given in Eq. (4). If local equilibrium positions $R_{0}(|\psi\rangle)$ exist, they satisfy the condition

$$
R_{0}^{4}\left(R_{0}-\delta\right)=\frac{3 d^{2}\langle\psi|W| \psi\rangle}{2 V_{0}},
$$

where $\langle\psi|W| \psi\rangle=\left\langle\psi\left|\left(j_{z} Z_{a} Z_{b}+j_{x} X_{a} X_{b}+j_{y} Y_{a} Y_{b}\right)\right| \psi\right\rangle$ are possible expectation values with respect to each of the four 
Bell states. Figure 1(b) shows the total potential landscape seen by particle $b$ for each of the spin Bell states, displaying the spin-dependent potentials. Note that the state $\left|\psi^{+}\right\rangle$ does not exhibit a minimum; this is not a problem provided that the protected logical qubit states do not involve $\left|\psi^{+}\right\rangle$.

In between the equilibrium points of the potential landscapes in Fig. 1(b) there are corrective sites, where devices that we call correctors are present. Correctors are represented in Fig. 1(a) as loops. The function of the corrective devices is to execute a unitary operation on the spin subspace once the particle approaches their site. There are two correctors, denoted $L 1$ and $L 2$. For an illustration of the device functioning, in the remainder of this section we treat the correctors $L 1$ and $L 2$ as qubits. Note, however, that there are a number of ways of implementing such devices and alternatives to the qubit model is discussed in the Sec. III.

Consider the $L 1$ device to have basis states $\left\{\left|\mu_{0}^{1}\right\rangle,\left|\mu_{1}^{1}\right\rangle\right\}$. Whenever the particle enters one of the $L 1$ loops, the unitary operation $Z_{b} X_{L 1}$ is executed, where $X_{L 1}=\left|\mu_{0}^{1}\right\rangle\left\langle\mu_{1}^{1}\right|+$ $\left|\mu_{1}^{1}\right\rangle\left\langle\mu_{0}^{1}\right|$. It is important that $L 1$ is insensitive to whether particle $b$ entered the innermost or outermost loop, since obtaining that information would collapse the spin state of the system as it is correlated with motion. On this note, we highlight the importance of choosing the equilibrium position of the potentials associated with $\left|\psi^{-}\right\rangle$and $\left|\phi^{-}\right\rangle$equidistant from the innermost and outermost loops of $L 1$. Note further that the position state of the atom is delocalized (e.g., as in the ground state of the corresponding harmonic motion). Hence, the arrival times of atom $b$ on either of the innermost or outermost loops are uncertain and provided that the potential landscapes are suitably adjusted, no information on the spin logical state can be obtained during the correction process. The $L 2$ system, or middle corrector, has basis states $\left\{\left|\mu_{0}^{2}\right\rangle,\left|\mu_{1}^{2}\right\rangle\right\}$ and whenever particle $b$ enters $L 2$, the unitary $X_{b} X_{L 2}$ is executed, where $X_{L 2}$ is once again the bit-flip operator on the corresponding basis states of $L 2$.

We have the following operations:

$$
L 1: Z_{b} X_{L 1}, \quad L 2: X_{b} X_{L 2} .
$$

Note that these unitaries act on the spins conditional on the position of the particle. Hence, when tracing out the position degree of freedom, the action of the corrective sites manifests as dissipative maps on the spin subspace.

The logical basis states of the nucleus are defined as

$$
\begin{aligned}
& |\overline{0}\rangle=\left|\psi^{-}\right\rangle, \\
& |\overline{1}\rangle=\left|\phi^{-}\right\rangle,
\end{aligned}
$$

and an arbitrary logical qubit state is

$$
|\Psi\rangle=\alpha|\overline{0}\rangle+\beta|\overline{1}\rangle
$$

TABLE I. The effect of physical errors on logical basis states and the final corrected state after action of the qubot.

\begin{tabular}{lllc}
\hline \hline Error & $\left|\psi^{-}\right\rangle$ & $\left|\phi^{-}\right\rangle$ & Corrected state \\
\hline$X_{a}$ & $-\left|\phi^{-}\right\rangle$ & $-\left|\psi^{-}\right\rangle$ & $-\alpha|\overline{0}\rangle-\beta|\overline{1}\rangle$ \\
$X_{b}$ & $+\left|\phi^{-}\right\rangle$ & $+\left|\psi^{-}\right\rangle$ & $\alpha|\overline{0}\rangle+\beta|\overline{1}\rangle$ \\
$Z_{a}$ & $+\left|\psi^{+}\right\rangle$ & $+\left|\phi^{+}\right\rangle$ & $\alpha|\overline{0}\rangle-\beta|\overline{1}\rangle$ \\
$Z_{b}$ & $-\left|\psi^{+}\right\rangle$ & $+\left|\phi^{+}\right\rangle$ & $-\alpha|\overline{0}\rangle-\beta|\overline{1}\rangle$ \\
$Z_{a} X_{a}$ & $-\left|\phi^{+}\right\rangle$ & $-\left|\psi^{+}\right\rangle$ & $\alpha|\overline{0}\rangle-\beta|\overline{1}\rangle$ \\
$Z_{b} X_{b}$ & $+\left|\phi^{+}\right\rangle$ & $-\left|\psi^{+}\right\rangle$ & $-\alpha|\overline{0}\rangle-\beta|\overline{1}\rangle$ \\
\hline \hline
\end{tabular}

Note that a superposition of the $|\overline{0}\rangle,|\overline{1}\rangle$ states implies that particle $b$ is in a superposition of singlet and triplet spin states, implying a superposition of different spatial equilibrium points.

To understand how the qubot delays decoherence and partially protects the logical qubit, we must follow carefully what happens to the particles when a physical error occurs in one of the spins. Single-physical-qubit errors are assumed to be much more likely than multiqubit errors [35] and the depolarizing channel is considered as a decoherence model. A summary of the possible errors and how they act on logical basis states is shown in Table I.

As an illustration, consider the example of a bit-flip in the first spin described by the $X_{a}$ operator. Initially, an arbitrary logical qubit state $|\Psi\rangle=\alpha\left|\psi^{-}\right\rangle+\beta\left|\phi^{-}\right\rangle$is in a superposition of equilibrium positions $R_{0}\left(\left|\psi^{-}\right\rangle\right)$and $R_{0}\left(\left|\phi^{-}\right\rangle\right)$given by solutions of Eq. (9). The $X_{a}$ error changes the spin state of the particles according to

$$
\alpha\left|\psi^{-}\right\rangle+\beta\left|\phi^{-}\right\rangle \rightarrow-\alpha\left|\phi^{-}\right\rangle-\beta\left|\psi^{-}\right\rangle,
$$

and hence the interaction potential of the particles is changed accordingly. After the error, the possible positions of particle $b$ are no longer equilibrium points of the potential landscapes. For the case in which $b$ is initially at $R_{0}\left(\left|\psi^{-}\right\rangle\right)$, the particles repel, forcing $b$ into $L 2$. Similarly, for $R_{0}\left(\left|\phi^{-}\right\rangle\right)$, occurrence of the error causes an attractive force that pulls $b$ into $L 2$. Once $b$ reaches the loop, the operator $X_{b} X_{L 2}$ is applied, restoring the logical qubit to the original state and driving the system back to the initial superposition of equilibrium points. For an illustration of the qubot operation cycle, see Fig. 2. Naturally, this process introduces kinetic energy in the form of phonons, which must be removed if particle $b$ is to settle back into the original state. This implies the need for a dissipative force acting on $b$, which could be provided by state-independent cooling of the atom motion. For now, we assume that such cooling is present and this phonon issue is discussed further in the Sec. III.

Similar processes occur for $X_{b}$ and $Z_{b}$ errors: a combination of spin-motion dynamics and subsequent application of the loop operators corrects errors and restores the system to the initial arbitrary logical state. The qubot is also 
able to correct a concatenation of phase and bit-flip errors, given by $Y_{b}$. Note that this requires a passage through two correctors.

The present qubot model is not able to correct all errors. As can be seen in Table I, logical basis states transform under $Z_{a}$ with opposite parity, thus inducing a phase error in the logical qubit. This imparts on the $Y_{a}$ error, since $i Y_{a}=Z_{a} X_{a}$. This imperfection can be traced back to the fact that the qubot uses two physical qubits to encode a logical state. The quantum Hamming bound [46] implies that for single-qubit errors, a minimum of five qubits are required to achieve complete fault tolerance for one logical qubit. Despite this partial fault tolerance, the qubot can delay decoherence of arbitrary logical qubit states and for some specific states it is even able to preserve it regardless of the error - as, for example, the singlet $|\psi\rangle=\left|\psi^{-}\right\rangle$. More general models implementing perfect quantum errorcorrecting codes [47] can nevertheless be devised at the expense of more particles or higher spin states. Note that to protect arbitrary logical qubit states, the qubot potential landscapes must distinguish between all the four elements of the Bell basis, as in Fig. 1(b). If the landscape for two or more Bell states is indistinguishable, certain errors will not cause any effect upon the atom, preventing the action of the correctors. Note also that the order of the potential minima for each Bell state defines the choice of position and action for the corrective sites, as well as the choice of logical basis states.

It is instructive to consider the qubot operation under a depolarizing channel acting on particle $b$ alone. Let us denote the environment states as $\left|e_{j}\right\rangle$. Decoherence causes the joint particle-environment-corrector state to evolve according to

$$
\begin{aligned}
& |\Psi\rangle\left|e_{0}\right\rangle\left|\mu_{0}^{1} \mu_{0}^{2}\right\rangle \rightarrow \sqrt{1-p}\left(\alpha\left|\psi^{-}\right\rangle+\beta\left|\phi^{-}\right\rangle\right)\left|e_{0}\right\rangle\left|\mu_{0}^{1} \mu_{0}^{2}\right\rangle \\
& \quad+\sqrt{\frac{p}{3}}\left(\alpha\left|\phi^{-}\right\rangle+\beta\left|\psi^{-}\right\rangle\right)\left|e_{1}\right\rangle\left|\mu_{0}^{1} \mu_{0}^{2}\right\rangle \\
& \quad+\sqrt{\frac{p}{3}}\left(-\alpha\left|\psi^{+}\right\rangle+\beta\left|\phi^{+}\right\rangle\right)\left|e_{2}\right\rangle\left|\mu_{0}^{1} \mu_{0}^{2}\right\rangle \\
& \quad+\sqrt{\frac{p}{3}}\left(\alpha\left|\phi^{+}\right\rangle-\beta\left|\psi^{+}\right\rangle\right)\left|e_{3}\right\rangle\left|\mu_{0}^{1} \mu_{0}^{2}\right\rangle
\end{aligned}
$$

where $p$ denotes the error probability. Equation (15) describes the depolarizing dynamics suffered by the logical qubit, with the first term proportional to $\sqrt{1-p}$ corresponding to no decoherence and the subsequent terms proportional to $\sqrt{p / 3}$ corresponding to errors on the logical qubit. Note that at this stage, the corrective devices remain unaffected while the system undergoes errors and the environment learns when an error has occurred. Tracing out the environment, the above evolution induces a dissipative map on the spin system, increasing its entropy and causing decoherence of the original state.
With the occurrence of errors, the potential landscapes acting on $b$ undergo a change, forcing the action of the correctors upon the spin state of the nucleus. Purity of the logical qubit is restored at the expense of an increase in entropy for the correctors; after a correction event, Eq. (15) evolves to

$$
\begin{gathered}
|\Psi\rangle\left(\sqrt{1-p}\left|e_{0}\right\rangle\left|\mu_{0}^{1} \mu_{0}^{2}\right\rangle+\sqrt{\frac{p}{3}}\left|e_{1}\right\rangle\left|\mu_{0}^{1} \mu_{1}^{2}\right\rangle\right. \\
\left.-\sqrt{\frac{p}{3}}\left|e_{2}\right\rangle\left|\mu_{1}^{1} \mu_{0}^{2}\right\rangle-\sqrt{\frac{p}{3}}\left|e_{3}\right\rangle\left|\mu_{1}^{1} \mu_{1}^{2}\right\rangle\right),
\end{gathered}
$$

where we can see that the original logical qubit state is restored and the environment becomes correlated with the states of the correctors. After a single error correction, the states of the correctors must be reset to the pure initial state $\left|\mu_{0}^{1} \mu_{0}^{2}\right\rangle$. This is a nonunitary operation that requires energy expenditure, similar to erasing a quantum state $[48,49]$, and it can be implemented as a nonequilibrium stochastic process. This corresponds to a consumption of resources by the qubot analogous to the consumption of resources by biological molecular machines and living organisms. The energetic costs of quantum operations have been analyzed [50-52] and experimentally demonstrated [53-55]. Irrespective of the physical implementation of the corrective sites, such consumption of resources is a mandatory part of the qubot operation in accordance to the laws of thermodynamics.

\section{IMPLEMENTATION}

\section{A. Potential engineering}

Spin-spin interactions of the form given in Eq. (2) suitable for implementing quantum robots could be engineered in a number of different atomic and molecular systems. In this section, a physical implementation using laser-dressed Rydberg atoms $[42,43,56]$ is discussed. As will be shown, instances of the qubot described in the previous section can be realized for realistic experimental parameters, provided that we choose the correct logical basis elements and position of corrective sites. We focus on a qubot that stabilizes an effective entangled spin state against a depolarizing environment similar to the one outlined in Ref. [18]. We refer to this device as an entanglement qubot.

A pair of ${ }^{87} \mathrm{Rb}$ atoms labeled $a$ and $b$ constitute the qubot nucleus. Effective spin states are provided by hyperfine levels of $b$, specifically

$$
\begin{aligned}
& |0\rangle=\left|5^{2} S_{1 / 2}, F=1, m_{F}=1\right\rangle, \\
& |1\rangle=\left|5^{2} S_{1 / 2}, F=2, m_{F}=2\right\rangle,
\end{aligned}
$$

with energy difference $\omega_{01}$. The atom-atom interaction potential is induced by dressing the $|0\rangle,|1\rangle$ states with 
(a)

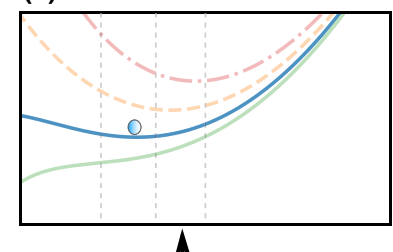

(d)

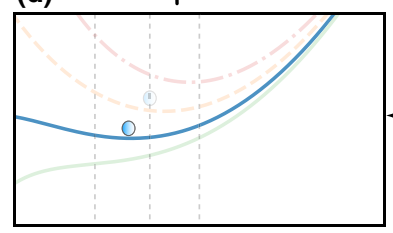

(b)

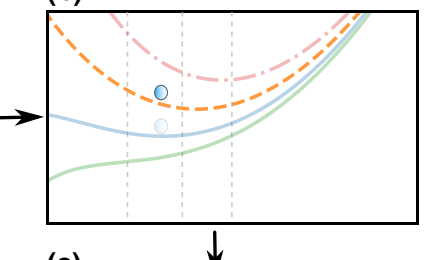

(c)

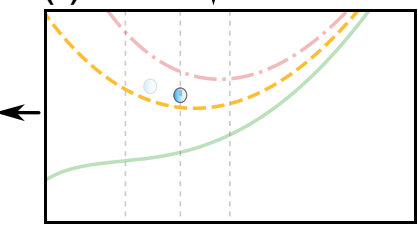

FIG. 2. An example of a qubot cycle: (a) particle $b$ rests in its equilibrium position, while the spin states form a singlet $|\overline{0}\rangle=$ $\left|\psi^{-}\right\rangle$; (b) an error occurs, changing the potential landscape seen by $b$; (c) the particle is forced into loop $L 2$, which restores the original spin state; and (d) the particle goes back to the original equilibrium position.

two strongly interacting Rydberg Zeeman sublevels in the $n^{2} P_{1 / 2}$ manifold via Rabi oscillations with detunings $\Delta_{ \pm}$ and frequencies $\Omega_{ \pm}$, using $\sigma^{ \pm}$-polarized light. The interaction between Rydberg states arises from a van der Waals potential of the form $C_{6} R^{-6}$ and a fixed orientation of the two particles is considered, with the atoms polarized perpendicular to the plane. Large detunings guarantee that only a small fraction of the Rydberg states is admixed to the $|0\rangle,|1\rangle$ levels while maintaining a long lifetime. Following Ref. [42], the Rydberg states are

$$
\left|r_{ \pm}\right\rangle=\left|n^{2} P_{1 / 2}, m_{j}= \pm 1 / 2\right\rangle\left|m_{I}=3 / 2\right\rangle,
$$

with an energy difference $\Delta E_{r}$. Detunings are chosen such that the energy-conservation condition $\Delta E_{r}=\left(\Delta_{+}-\Delta_{-}\right)$ is satisfied. A level diagram is shown in Fig. 3. The atoms are trapped in one-dimensional (1D) potentials, insensitive to their internal states. State-independent trapping of Rydberg-dressed atoms can be achieved in so-called magic [57,58] and magnetic traps [59]. While atom $a$ is fixed at the origin, $b$ is able to move under the influence of a force resulting from the combination of an external tweezer and the atom-atom interaction potential.

As in quantum chemistry $[19,20]$, the time scale associated with electronic dynamics is much shorter than the time scale of nuclei motion. An effective spin-dependent Born-Oppenheimer potential can therefore be derived at fixed atomic separations $R$. In the limit of large detunings $\Omega_{ \pm} \ll \Delta_{ \pm}$and for $\Delta_{+} / \Delta_{-}<0, \Delta_{+}+\Delta_{-}<0$, adiabatic elimination [60] can be used in the rotating frame to obtain an effective interaction acting on the subspace generated by the $|0\rangle,|1\rangle$ states to fourth order in $\Omega_{ \pm} / \Delta_{ \pm}$,

$$
V_{I}(R)=J_{z} Z_{a} Z_{b}+J_{x} X_{a} X_{b}+J_{y} Y_{a} Y_{b}+J_{\|}\left(Z_{a}+Z_{b}\right)
$$

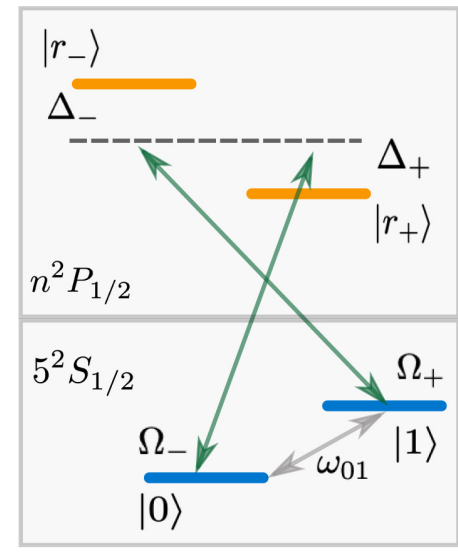

FIG. 3. The level schematics for the entanglement qubot.

where $J_{\alpha}(R)(\alpha=x, y, z)$ are radial steplike coefficients depending on the Rabi frequencies $\Omega_{ \pm}$, detunings $\Delta_{ \pm}$and van der Waals $C_{6}$ coefficients for the $n^{2} P_{1 / 2}$ manifold. $J_{\|}$is an effective magnetic field, which we assume can be canceled by an additional weak nonhomogeneous field on the order of $2 \mathrm{G}$ (for explicit definitions, formulas, and details on the potential and effective magnetic field, respectively, see Appendices A and B).

A plot of the $J_{\alpha}$ spin pattern for $n=60$, detunings $\Delta_{-}=-\Delta_{+}=2 \pi \times 50 \mathrm{MHz}$ and Rabi frequencies $\Omega_{-}=$ $\Omega_{+} / 3=2 \pi \times 3 \mathrm{MHz}$ can be seen in Fig. 4(a). Note that these are in the same parameter region as used for realizing the quantum spin ice Hamiltonian on a kagome lattice in Ref. $[42,61]$. The parameters defining a qubot potential are not unique, allowing some freedom in the construction (for an example of a different set of numbers and the resulting spin pattern, see Appendix C).

From the spin-pattern coefficients together with Eqs. (5)-(8) and a trap potential $V_{t}(R)$, we can derive the collective spin-dependent potentials acting on particle $b$. Consider a trap potential provided by two neighboring optical tweezers,

$$
V_{t}(R)=V_{0}\left[\left(R-\delta_{1}\right)^{2}+\left(R-\delta_{2}\right)^{2}\right]
$$

where $V_{0}=15 \mathrm{kHz} / \mu \mathrm{m}^{2}, \delta_{1}=1.6 \mu \mathrm{m}$, and $\delta_{2}=2.0 \mu \mathrm{m}$. The resulting spin-dependent potential landscapes $V(R)$ can be seen in Fig. 4(b), where each trace corresponds to a different Bell state of the two atoms. Note that the equilibrium positions are separated by approximately $0.3 \mu \mathrm{m}$. The trap frequencies are approximately $\omega_{t} / 2 \pi \approx 1 \mathrm{kHz}$. Possible positions for the corrective sites $L 1$ and $L 2$, corresponding to the transformations given in Eq. (10), are represented by dashed vertical lines. Note the potential landscapes for the Bell states $\left|\psi^{-}\right\rangle$and $\left|\psi^{+}\right\rangle$overlap. This implies that one cannot choose either $\left|\psi^{-}\right\rangle$or $\left|\psi^{+}\right\rangle$as protected states, as in this case, phase errors could not be corrected. The protected logical state is chosen to be $\left|\phi^{+}\right\rangle$. 
(a)

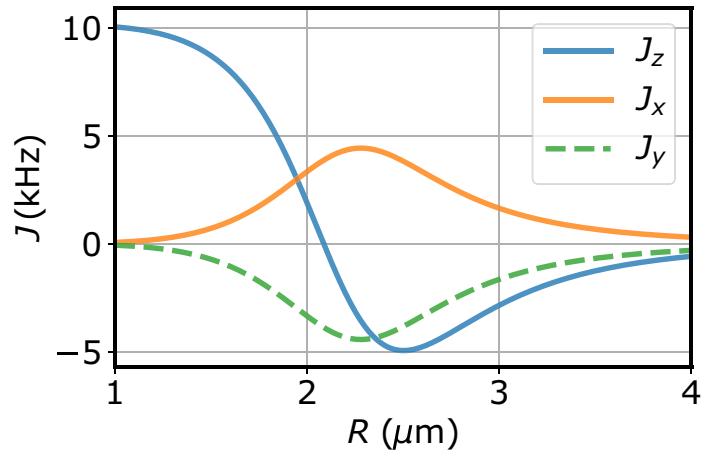

(b)

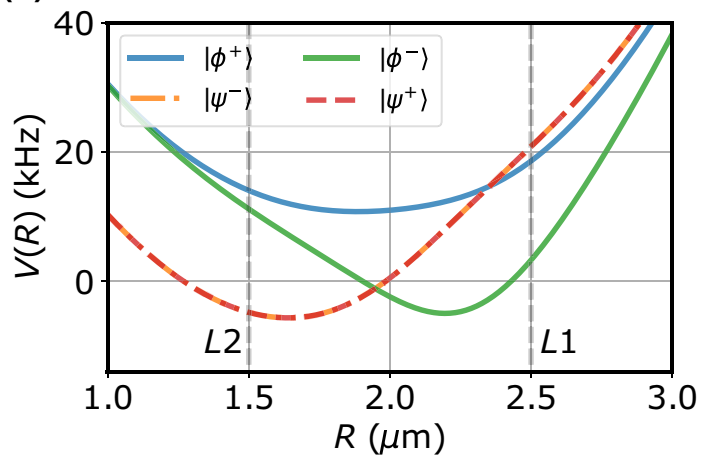

FIG. 4. (a) The spin pattern, corresponding to the coefficients of Eq. (20), for the parameters $n=60, \Delta_{-}=-\Delta_{+}=2 \pi \times 50 \mathrm{MHz}$ and $\Omega_{-}=\Omega_{+} / 3=2 \pi \times 3 \mathrm{MHz}$. (b) Collective spin-dependent potential landscapes. Each trace corresponds to a Bell state of the qubot nucleus. Corrective devices $L 1$ and $L 2$ are positioned outside the potential minima as, for example, the dashed vertical lines indicate.

\section{B. Corrective sites}

Correctors $L 1$ and $L 2$ have previously been considered to be qubits acting as an entropy sink for maintaining the purity of the protected logical qubit state carried by the nucleus. The interaction between superconducting quantum electronics and atomic [62], molecular [63], and mesoscopic particles [64] has been extensively studied in the context of hybrid quantum systems and the coupling between $\mathrm{N}-V$ centers and superconductors has been observed [65]. A number of different implementations involving superconducting qubit systems are therefore expected.

Beyond qubits, we may consider additional atoms as candidates for the implementation of corrective devices. Controlled atomic collisions [66] would provide the mechanism for position-dependent unitary operations. One could envision a lattice with arrays of data particles interpolated with corrective particles, analogous to the surface code [35]; the occurrence of errors would alter the interaction between the data particles, enabling or inhibiting motion and tunneling - and consequently interactions - with neighboring corrective sites. It would be like a surface code in motion, where errors induce controlled motion leading to correction feedback. It is important to stress that in the course of the qubot action, the entropy of the corrective atoms would increase and a dissipative map for restarting the correctors in their original state would have to be continuously enforced, for example, through an amplitude-damping channel [18].

Corrective devices could also be implemented using Rabi oscillations between the $|0\rangle,|1\rangle$ levels. By carefully tuning the Rabi frequency of the transition and the profile of the spin-dependent potentials in Fig. 4(b), it is in principle possible to engineer the transit time of atom $b$ through $L 1$ and $L 2$ such that $Z_{b}$ and $X_{b}$ operations are applied, analogous to the transit-time-stimulated decay in ammonia masers [67] and Ramsey interferometry in atomic fountain clocks [68]. In this implementation - probably the most practical from an experimental point of view - the electromagnetic field assumes the role of an entropy sink, since conditional $X$ and $Z$ operations on the atom would introduce uncertainties in the intensity and phase of the field, respectively. A schematics of this implementation is shown in Fig. 5.

\section{Operation, cooling, and lifetime}

Operation of the qubot proceeds as described in the previous section: the occurrence of an error induces a change in the potential landscape seen by atom $b$, thus forcing it into one of the corrective sites $L 1$ or $L 2$. Note that errors can occur due to external environmental influence or intrinsically due to thermal and quantum fluctuations of the atomic motion. Consider atom $b$ in a thermal state. For temperatures on the order of $10 \mathrm{nK}$, which are reachable for atomic ensembles [69], the occupation number of the atomic motion is $\bar{n} \approx 0.1$, highlighting the fact that the atom is effectively in the trap ground state. The zero-point motion of the atom is approximately $R_{\mathrm{ZPM}} \simeq \sqrt{\hbar / 2 m \omega_{t}} \approx$ $0.23 \mu \mathrm{m}$, indicating that at $10 \mathrm{nK}$, quantum fluctuations can cause the atom to reach the corrective sites even when no environmental error takes place, inducing change in the

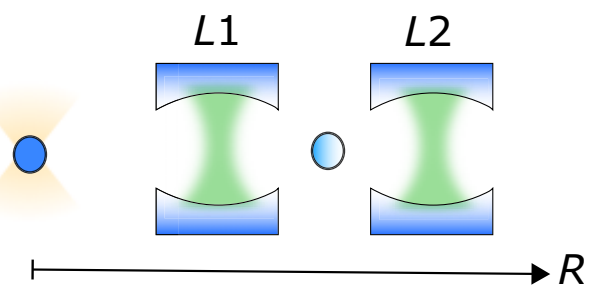

FIG. 5. Corrective sites as Rabi oscillations. 
qubot state. Hence, intrinsic fluctuation errors are expected to constitute a portion of the total errors. In the next section, a model of the qubot operation taking into account intrinsic and external errors is discussed.

Errors can be effectively corrected provided that the qubot nucleus undergoes constant cooling of its motional degrees of freedom to dissipate the kinetic energy gained by mechanical forces due to potential changes. Such cooling mechanism needs to preserve the quantum information stored in the nucleus, so it must be insensitive to the quantum state stored in the spins. State-insensitive cooling of neutral atoms can be achieved via superfluid immersion [70], cavity cooling [71], or sympathetic cooling through spin-independent Rydberg interactions with neighboring atoms [72].

What is the order of magnitude of the expected lifetime for the protected entangled state? The $60 P_{1 / 2}$ Rydberg state has a lifetime on the order of $\tau_{r} \approx 133 \mu \mathrm{s}$ [73]. This implies a bare lifetime for the effective spin state of $\tau_{s} \approx$ $\left(2 \Delta_{-} / \Omega_{-}\right)^{2} \tau_{r} \approx 9 \mathrm{~ms}$ [42], corresponding to a spin decoherence rate $\Gamma \approx 111 \mathrm{~Hz}$. A decay process to the ground state $|0\rangle$ is defined by the following transformations:

$$
\begin{gathered}
|0\rangle\left|e_{0}\right\rangle \rightarrow|0\rangle\left|e_{0}\right\rangle, \\
|1\rangle\left|e_{0}\right\rangle \rightarrow \sqrt{1-\tau_{s}^{-1} d t}|1\rangle\left|e_{0}\right\rangle+\sqrt{\tau_{s}^{-1} d t}|0\rangle\left|e_{1}\right\rangle,
\end{gathered}
$$

where the first ket corresponds to the spin of the particle while the second ket represents the environment state. The action of this quantum channel upon the elements of the Bell basis can be written in terms of strings of Pauli errors [74]. It is thus expected that the qubot is able to extend the lifetime of Rydberg-dressed entangled states.

\section{DYNAMICS SIMULATION}

Exploration of the qubot requires simulation of its errorcorrection dynamics. Any such simulation must take into account the effects of quantum fluctuations of atomic motion, as these fluctuations are in themselves a source of errors that can disturb the protected Bell state. A firstprinciples description of the spin and motion degrees of freedom is intricate, as the spin state is subject to transformations conditional on the motion state, which in itself is conditioned on the spin through the spin-dependent potential. As Wheeler would say [75]: spin tells matter how to move, matter tells spin how to turn.

To capture the essential features of the qubot, we propose an open quantum system model in which the motion and spin degrees of freedom follow a set of discrete-time coupled stochastic Schrödinger equations. Each realization of the evolution is described in terms of sequences of quantum state pairs, denoted $|\psi\rangle$ for the spin and $|\phi\rangle$ for the motion degree of freedom. Averaging over many realizations of the stochastic process results in the mean behavior of the system.

The spin and motion degrees of freedom act as environments for each other. This idea can be used to motivate the model as follows. For simplicity, discretize (1D) space into a set of points $R_{k}$. The position state reads

$$
|\phi\rangle=\sum_{k} \phi\left(R_{k}\right)\left|R_{k}\right\rangle
$$

where $\left|\phi\left(R_{k}\right)\right|^{2}$ gives the probability of finding the particle at position $R_{k}$. The initial state evolves in a small time increment $\delta t$ according to

$$
\begin{aligned}
& |\psi\rangle|\phi\rangle \stackrel{\delta t}{\rightarrow} \sum_{i} \phi\left(R_{i}\right)\left(T\left(R_{i}\right)|\psi\rangle\right)\left(W(|\psi\rangle)\left|R_{i}\right\rangle\right) \\
& \quad=|\Psi(t+\delta t)\rangle
\end{aligned}
$$

where $T\left(R_{i}\right)$ is the identity operator unless $R_{i}=R_{L 1}$ or $R_{i}=R_{L 2}$, for which

$$
\begin{aligned}
& T\left(R_{L 1}\right)=Z_{b}, \\
& T\left(R_{L 2}\right)=X_{b} .
\end{aligned}
$$

The operator $W(|\psi\rangle)$ contains information on the spindependent potential and is responsible for the evolution of the motion state. Expanding $|\Psi(t+d t)\rangle$,

$$
\begin{aligned}
|\Psi(t+\delta t)\rangle= & \sum_{i \neq L 1, L 2} \phi\left(R_{i}\right)|\psi\rangle\left[W(|\psi\rangle)\left|R_{i}\right\rangle\right] \\
& +\phi\left(R_{L 1}\right)\left(Z_{b}|\psi\rangle\right)\left[W(|\psi\rangle)\left|R_{L 1}\right\rangle\right] \\
& +\phi\left(R_{L 2}\right)\left(X_{b}|\psi\rangle\right)\left[W(|\psi\rangle)\left|R_{L 2}\right\rangle\right] .
\end{aligned}
$$

Note that this is an entangled state of the motion and internal spin degrees of freedom of the atoms. Since we are primarily interested in the collective spin state, we assume that the collective internal degrees of freedom of the atoms are continuously monitored in the Bell basis, causing the above state to continuously collapse to a random separable state, allowing the phase information and correlations of the global state to be ignored. This monitoring of the joint spin state in the Bell basis can be achieved by continuous measurement of the force acting on particle $a$, since the interaction between the particles is given by their joint spin state. Continuous position and force measurements on individual quantum systems have been previously demonstrated [76-78], highlighting the experimental feasibility of this assumption. Note further that under this monitoring assumption, we can describe the dynamics of the system within a simpler scenario and yet verify the errorcorrection capability of the proposed qubot. The motion 
state then acts as an environment for the spin, inducing corrective jump operators,

$$
\begin{aligned}
& L_{1}=\sqrt{\gamma_{L 1}} Z_{b}, \\
& L_{2}=\sqrt{\gamma_{L 2}} X_{b},
\end{aligned}
$$

where we define the correction rates as

$$
\begin{aligned}
& \gamma_{L 1} d t=\left|\phi\left(R_{L 1}\right)\right|^{2}, \\
& \gamma_{L 2} d t=\left|\phi\left(R_{L 2}\right)\right|^{2} .
\end{aligned}
$$

Note that the probability of a given corrective jump occurring is also the probability of finding the particle in the corresponding corrective site. In addition to corrective jumps, the spin state is also subject to the effect of a depolarizing channel due to an external decoherence environment, defined in terms of the collapse operators

$$
L_{3}=\sqrt{\frac{\Gamma}{3}} X_{b}, \quad L_{4}=\sqrt{\frac{\Gamma}{3}} Y_{b}, \quad L_{5}=\sqrt{\frac{\Gamma}{3}} Z_{b},
$$

where $\Gamma$ is the decoherence rate.

Conversely, spin acts as an environment to the motion state. If no spin-corrective jump occurs, the motion state is left almost unperturbed, according to Eq. (28), and evolves through the unitary predicted by the spin state $|\psi\rangle$ plus the effects of a damping collapse operator provided by an additional spin-insensitive cooling environment with damping rate $\kappa$ acting as a drain of kinetic energy, as discussed previously. Note that state-insensitive damping is an important requirement in atomic quantum simulators and has been experimentally demonstrated in Ref. [79], for example; in the particular case of dressed Rydberg interactions, state-independent cooling can be achieved via coupling of atom $b$ to ancillary atoms through engineered state-insensitive Rydberg potentials, as proposed in Ref. [72]. That scheme, which works by a process of phonon swapping between the data and ancillary qubits, is devised to be compatible with the effective interactions leading to Eq. (20) and allows for simultaneous cooling and the effective spin dynamics of the qubot. Moreover, if a corrective jump $L_{1}$ or $L_{2}$ happens, the motion state collapses to $\left|R_{L 1}\right\rangle$ or $\left|R_{L 2}\right\rangle$, respectively. The collapsed state subsequently evolves according to the unitary predicted by the spin state $|\psi\rangle$ plus the additional damping collapse operator. When spin jumps happen, the motion Hamiltonian must be updated accordingly for the next time iteration.

This evolution can be implemented via a coupled Monte Carlo method. First, define the motion Monte Carlo procedure (MMC) for a damped harmonic oscillator as follows:

(1) Define the motion state $|\phi\rangle$ and Hamiltonian $H$.

(2) Compute $\delta v=\kappa \delta t\left\langle\phi\left|a^{\dagger} a\right| \phi\right\rangle$.
(3) Choose a uniformly distributed random number $q \in$ $[0,1]$.

(4) If $q<\delta v$, update $|\phi\rangle \leftarrow a|\phi\rangle / \sqrt{\delta v / \delta t}$.

(5) If $q>\delta v$, update $|\phi\rangle \leftarrow e^{-i \hat{H} \delta t}|\phi\rangle / \sqrt{1-\delta v}$, where $\hat{H}=H-(i / 2) a^{\dagger} a$.

We denote by $M(|\phi\rangle, H, \delta t)$ the output of the above procedure for input state $|\phi\rangle$ and Hamiltonian $H$, over a time step $\delta t$. This output consists of the updated motion state after one time step.

The following algorithm, dubbed spin-motion Monte Carlo (SMMC), summarizes one-time iteration of the qubot dynamics:

(1) Define (update) motion and spin states $|\phi\rangle$ and $|\psi\rangle$ and motion Hamiltonian $H=H(|\psi\rangle)$.

(2) Define the correction rates $\gamma_{L 1} \delta t=\left|\left\langle R_{L 1} \mid \phi\right\rangle\right|^{2}, \gamma_{L 2} \delta t$ $=\left|\left\langle R_{L 2} \mid \phi\right\rangle\right|^{2}$, where $\delta t$ is the discrete time increment.

(3) Compute $\delta p_{k}=\delta t\left\langle\psi\left|L_{k}^{\dagger} L_{k}\right| \psi\right\rangle$ and $\delta p=\sum_{k} \delta p_{k}$.

(4) Choose a uniformly distributed random number $r \in$ $[0,1]$.

(5) If $r<\delta p$, update $|\psi\rangle \leftarrow L_{k}|\psi\rangle / \sqrt{d p_{k} / \delta t}$ with probability $\delta p_{k} / \delta p$.

(5.1) If jumps $L_{k}$ with $k=1$ or 2 occur, update $|\phi\rangle \leftarrow$ $\left|R_{L_{k}}\right\rangle$ and run $M\left(\left|R_{L_{k}}\right\rangle, H, \delta t\right)$. After MMC, update the motion state and the motion Hamiltonian to $H=H\left(L_{k}|\psi\rangle\right)$.

(5.2) If jumps $L_{k}$ with $k=3,4$ or 5 occur, run $M(|\phi\rangle, H, \delta t)$. After MMC, update the motion state and the motion Hamiltonian to $H=$ $H\left(L_{k}|\psi\rangle\right)$.

(6) If $r>\delta p$, update $|\psi\rangle \leftarrow e^{-i H_{s} \delta t}|\psi\rangle / \sqrt{1-\delta p}$, where $H_{s}=-i \sum_{k} L_{k}^{\dagger} L_{k}$.

(6.1) Run $M(|\phi\rangle, H, \delta t)$. After $\mathrm{MMC}$, update the motion state and the motion Hamiltonian to $H=H(|\psi\rangle)$.

(7) Go to step (1) for the next iteration.

A time series of quantum states $\{|\psi(t)\rangle,|\phi(t)\rangle\}$ is called a quantum trajectory of the system and can be obtained by iterating SMMC. The mean behavior of the qubot can be obtained by averaging the quantities of interest over many quantum trajectories. For example, we can define the overlap between the qubot spin state and the protected Bell state as $F=\mathbb{E}\left[\left|\left\langle\psi(t) \mid \phi^{+}\right\rangle\right|^{2}\right]$, where $\mathbb{E}[\cdots]$ denotes the ensemble average over all quantum trajectories. The quantity $F$ then measures how close the qubot spin state is, on average, to the protected state and hence quantifies how well the qubot functions.

To simplify the dynamics simulation, spin-dependent potentials are taken to be harmonic traps of equal 
resonance frequency. This removes any issues due to anharmonicity in the potentials and allows for the definition of fixed phonon creation and annihilation operators. The potentials shown in Fig. 4(b) are approximated as

$$
V(|\psi\rangle, R)=\frac{m \omega_{t}^{2}}{2}\left[R-R_{0}(|\psi\rangle)\right]^{2}
$$

where $\omega_{t} / 2 \pi=1 \mathrm{kHz}$ and the trap position $R_{0}(|\psi\rangle)$ is given by

$$
R_{0}(|\psi\rangle)= \begin{cases}R_{01}, & \text { if }\left|\phi^{+}\right\rangle, \\ R_{10}, & \text { if }\left|\phi^{-}\right\rangle \\ R_{00}, & \text { if }\left|\psi^{ \pm}\right\rangle\end{cases}
$$

The positions $R_{\alpha \beta}$ are dependent on the details of the experimental implementation. Inspired by Fig. 4(b), we consider $R_{01}=1.90 \mu \mathrm{m}, R_{10}=2.20 \mu \mathrm{m}$, and $R_{00}=1.64 \mu \mathrm{m}$. Since the Hamiltonian always appears inside a commutator, constant terms can be neglected without affecting the dynamics. Defining the origin of our reference frame at the minimum of the potential $V\left(\left|\phi^{+}\right\rangle\right)$and neglecting constant shifts, the Hamiltonian reads

$$
H(|\psi\rangle)=\omega_{t} a^{\dagger} a-m \omega_{t}^{2} \Delta R_{0}(|\psi\rangle) R_{\mathrm{ZPM}}\left(a^{\dagger}+a\right)
$$

where $a^{\dagger}, a$ are the creation and annihilation operators for the $\left|\phi^{+}\right\rangle$potential, given by

$$
\begin{aligned}
a & =\sqrt{\frac{m \omega_{t}}{2}}\left(R+\frac{i}{m \omega_{t}} P\right) \\
a^{\dagger} & =\sqrt{\frac{m \omega_{t}}{2}}\left(R-\frac{i}{m \omega_{t}} P\right),
\end{aligned}
$$

where $R$ and $P$ are the atom position and momentum operators of particle $b$, respectively, $R_{\text {ZPM }}$ is the corresponding zero-point motion, and $\Delta R_{0}(|\psi\rangle)=R_{0}(|\psi\rangle)-R_{0}\left(\left|\phi^{+}\right\rangle\right)$. The effect of a change in the spin state can be interpreted as the appearance of an additional force acting on particle $b$.

Figure 6 shows the result of iterating SMMC averaged over $10^{3}$ quantum trajectories, implemented using QuTiP [80], for the initial Bell-position state $\left|\phi^{+}\right\rangle|\chi\rangle$, where $|\chi\rangle$ is a Gaussian wave packet in position with uncertainty $\Delta R$ (for details of the parameters used in the simulation, see the figure caption). The top graph shows the mean overlap $F=\mathbb{E}\left[\left|\left\langle\psi(t) \mid \phi^{+}\right\rangle\right|^{2}\right]$ as a function of time for the qubot (thick green line) compared to the depolarizing channel alone (thin purple line). We can see that initially the qubot overlap drops faster than the free spins but it stabilizes at about $70 \%$, while free decohering spins decrease significantly below. The middle plot shows the atom position and its quantum uncertainty as a function of time: the action of the qubot stabilizes the location of the atom. Note that motion of the atom toward one corrective site is expected

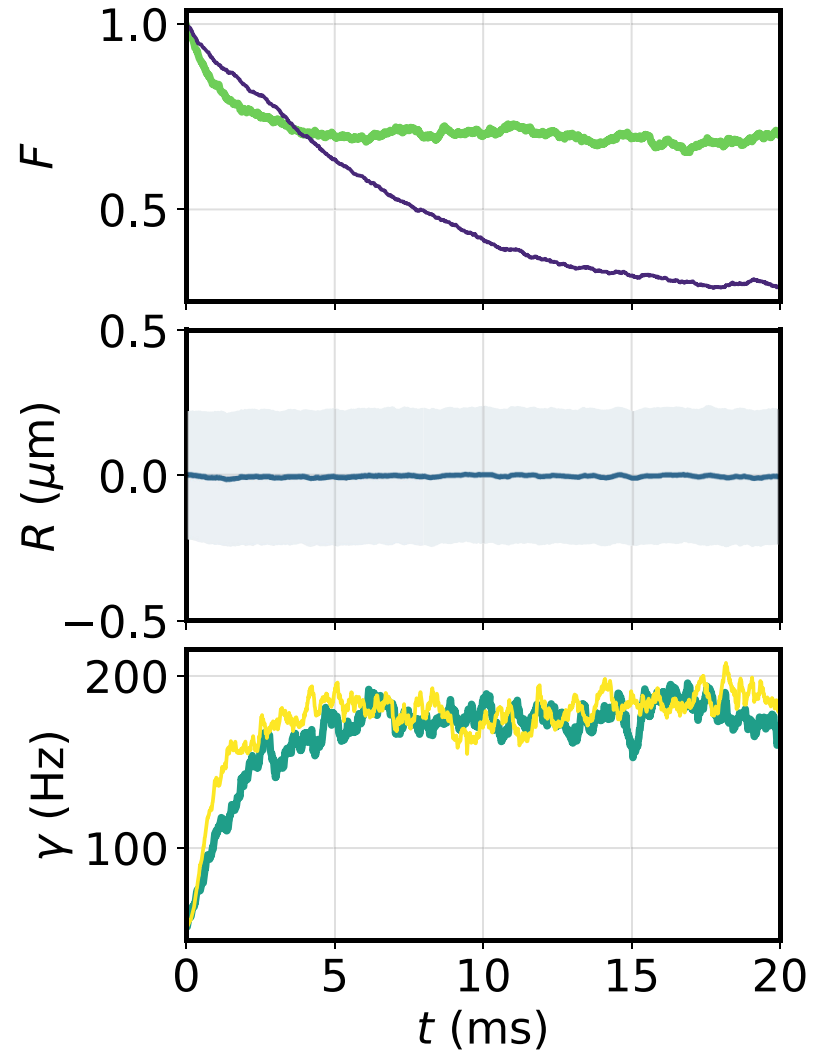

FIG. 6. The coupled spin-motion Monte Carlo simulation of the qubot, $10^{3}$ quantum trajectories. Top: average fidelity to the $\left|\phi^{+}\right\rangle$Bell state as a function of time for the qubot plus a depolarizing channel (thick green line) compared to the action of a depolarizing channel alone (thin purple line). Middle: the average position of the atom with corresponding quantum uncertainty (light blue shade). Bottom: the average correction rates $\gamma_{L 1}$ (light yellow line) and $\gamma_{L 2}$ (thick green line). The parameters used in the plot are as follows: decoherence rate $\Gamma=100 \mathrm{~Hz}$, trap frequency $\omega_{t}=1 \mathrm{kHz}$, damping rate $\kappa=0.1 \mathrm{~ms} \times \omega_{t}^{2}$, initial wave-packet uncertainty $\Delta R=0.22 \mu \mathrm{m}, R_{L 2}=-R_{L 1}=0.63 \mu \mathrm{m}$.

to increase the correction rates of that site and decrease the correction rates of the other site. This behavior can be seen in the bottom graph, where the rates are shown as a function of time. As expected, $\gamma_{L 1}$ (light yellow line) displays significant anticorrelation with $\gamma_{L 2}$ (thick green line).

The effect of finite temperature can be evaluated by adapting SMMC to include the motion-collapse operators $\sqrt{\kappa(\bar{n}+1)} a$ and $\sqrt{\kappa \bar{n}} a^{\dagger}$, representing contact with a thermal bath of phonons at temperature $T$ with coupling $\kappa$ and thermal occupation number $\bar{n}$, where $\bar{n}=1 /\left(e^{\hbar \omega_{t} / k_{B} T}-1\right)$. When in contact with a thermal bath, the particle initially in the ground state evolves to a thermal state with mean number of phonons $\bar{n}$, increasing the position spread and consequently the intrinsic qubot error rate. The spin overlap is thus expected to decrease with temperature.

The time-averaged steady-state overlap $\langle F\rangle_{s}$ as a function of temperature is plotted in Fig. 7. Each point is the result of time averaging $10^{2}$ quantum trajectories, 


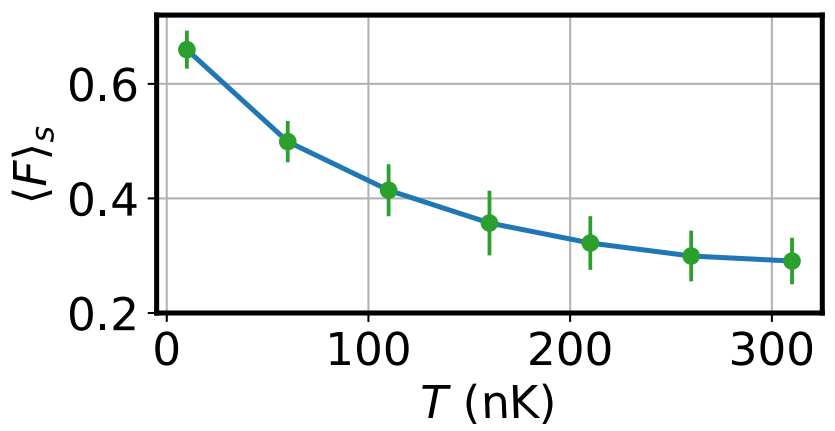

FIG. 7. The effect of contact with a thermal bath at temperature $T$ upon the steady-state time-averaged overlap $\langle F\rangle_{s}$. The time average is considered to start at $10 \mathrm{~ms}$, when the overlap has already achieved its steady value. The error bars correspond to one standard deviation. Each point is evaluated from $10^{2}$ quantum trajectories. The coupling to the heat bath is $\kappa=0.1 \mathrm{~ms} \times$ $\omega_{t}^{2}$ and all remaining parameters are the same as in Fig. 6.

with error bars corresponding to one standard deviation. As expected, the effect of contact with a heat bath is to decrease the overlap.

Quantum fluctuations of the atomic motion can induce internal errors if the atom interacts with the correctors when no external (decoherence) error has taken place. To quantify that effect, the steady-state overlap $\langle F\rangle_{s}$ and correction rates $\langle\gamma\rangle_{s}$ are numerically calculated for different values of the $L 1$ position $\left|R_{L 1}\right|$, as shown in Fig. $8 ; R_{L 1}=$ $-R_{L 2}$ is assumed. Note that if the correctors are too close to the equilibrium position of $\left|\phi^{+}\right\rangle\left(\left|R_{L 1}\right|<0.40 \mu \mathrm{m}\right)$, the steady-state overlap $\langle F\rangle_{s}$ falls below $50 \%$, while the mean rates for "correction" events are on the order of $1 \mathrm{kHz}$, due to the atom fluctuating toward $L 1$ or $L 2$ even in the absence of an error. As $\left|R_{L 1}\right|$ is increased, the steady-state overlap increases, reaching a maximum value $\langle F\rangle_{s} \approx 0.7$ for $\left|R_{L 1}\right| \approx 0.63$, and then decreases again as the correctors are placed further apart from the atom. The mean correction rates can be seen to decrease as the position $\left|R_{L 1}\right|$ is further increased, which is intuitive, since larger distances imply longer correction times. The optimal operation point $\left|R_{L 1}\right| \approx 0.63$ is such that the mean correction rates $\langle\gamma\rangle$ are of the same order as the decoherence rate $\Gamma=100 \mathrm{~Hz}$ (for more details, see Appendix D).

\section{DISCUSSION AND CONCLUSIONS}

Throughout this work, we discuss quantum robots, devices such as the one conceptualized in Ref. [18], capable of harnessing interactions between its constituent parts and the surrounding environment to achieve targeted tasks such as state protection against decoherence. We introduce for the first time a model of a qubot capable of partially protecting an arbitrary logical qubit state against general single-physical-qubit errors. The first physical implementation of an instance of such device, capable of protecting

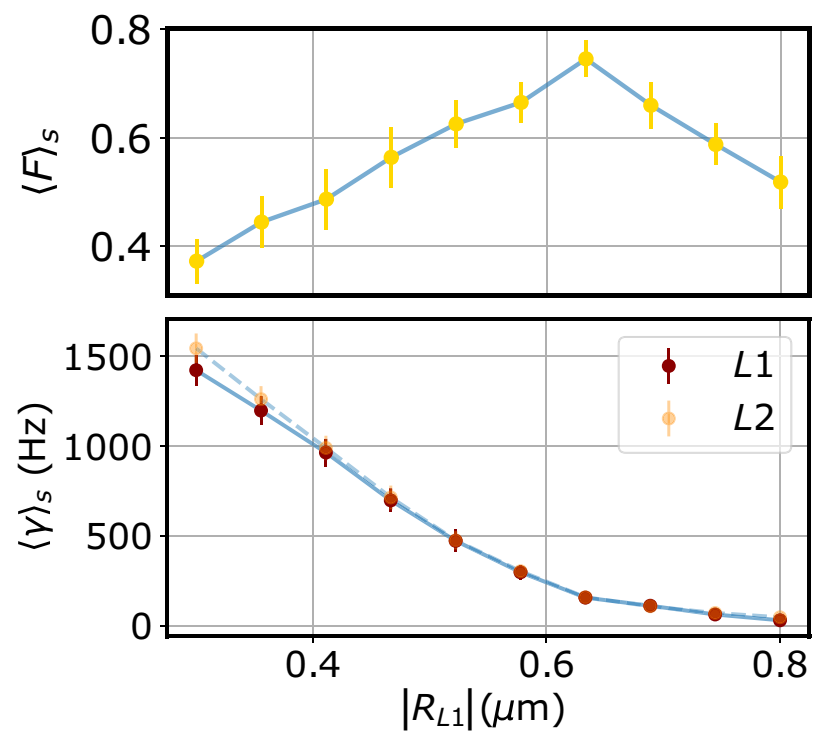

FIG. 8. The influence of corrector positioning. Top: steadystate overlap. Bottom: mean correction rates. The averages are considered from $10 \mathrm{~ms}$ onward, when the device is well settled in the steady state. The error bars correspond to one standard deviation. Each point is evaluated from $10^{2}$ quantum trajectories. All remaining parameters are the same as in Fig. 6.

a Bell state against the detrimental action of a depolarizing environment, is described, as well as Monte Carlo simulations of the qubot dynamics and the inclusion of effects due to contact of the device with a thermal bath.

From where we stand, several directions for future exploration can be sighted. For instance, a more thorough investigation of the capabilities of the proposed entanglement qubot remains to be done: by tuning the relevant parameters such as the Rydberg-level detunings $\Delta_{ \pm}$and trap potential $V_{t}(R)$, can we engineer a qubot capable of protecting entangled states other than the $\left|\phi^{+}\right\rangle$state? What about implementing a system analogous to the conceptual model, capable of protecting an arbitrary logical qubit? Could we extend the device to handle multiple qubits? Would the protection work against general physical errors? We focus on implementation using Rydberg-dressed atoms but that is certainly not the only possibility. What other opportunities are offered by considering different physical setups for qubots? Polar molecules provide a promising platform $[19,36,44,45]$, with the possibility of coupling to superconducting quantum electronics [63].

Synthetic molecular machines are one of the frontiers of nanotechnology $[6,8,9,21]$. Enabled by the idea of a quantum robot, we can envision extensions of the molecular machinery toolbox where the quantum states of the nanomachines play a fundamental role in their dynamics. These devices would combine resources from the environment, stochasticity, and nonequilibrium to execute coupled quantum motion and processing of quantum information 
entering the realm of quantum nanomechanics. For example, in the entanglement qubot, we could set the correction sites to perform the operation $L 1=L 2=X_{b}$, and initiate the spins in the state $\left|\psi^{+}\right\rangle$. This would cause a periodic spin-driven motion of the atom. It would be interesting to investigate the possibility of building quantum time crystals $[38,81,82]$ using this scheme.

Quantum robots with no moving parts are also a hitherto unexplored direction. In such devices, an error in one degree of freedom would unleash a chain of reactions in other internal nonmechanical parts of the system, which would act back on the affected degree of freedom and steer it to a desired state. This touches upon the theoretical issue of quantum feedback $[83,84]$, in a situation where the feedback itself is carried by quantum-mechanical information, rather than the usual classical information scheme in which a measurement result is used to guide a counteraction on the system.

Finally, a very intriguing thought is the combination of a large number of quantum robots interacting with each other. Large numbers of interacting classical active agents display fascinating emergent behavior $[85,86]$. Ensembles of active quantum agents, on the other hand, remain unexplored. Qubots offer a concrete path toward experimentally uncovering the physics of quantum active matter.

\section{ACKNOWLEDGMENTS}

I thank Bruno Melo, Lucianno Defaveri, Bruno Suassuna, Igor Brandão, and George Svetlichny for useful discussions and feedback on the manuscript. This work was financed in part by the Coordenacão de Aperfeiçoamento de Pessoal de Nível Superior-Brasil (CAPES) -Finance Code 001, the Conselho Nacional de Desenvolvimento Científico e Tecnológico (CNPq), and the FAPERJ Scholarship No. E-26/202.830/2019.

\section{APPENDIX A: EFFECTIVE POTENTIALS}

As described in the main text, the admixture of strongly interacting Rydberg states from the $n^{2} P_{1 / 2}$ manifold to the low-lying $5^{2} S_{1 / 2}$ Zeeman sublevels induces spatially dependent spin-spin interactions of the form given in Eq. (20). For completeness, we reproduce the main results of Ref. [42], outlining the toolbox for engineering a wide range of effective spin interactions.

The interaction coefficients $J_{\alpha}$ are calculated by adiabatic elimination of the Rydberg levels $\left|r_{ \pm}\right\rangle$up to fourth order in $\Delta / \Omega$ and are given by

$$
\begin{aligned}
& J_{z}(R)=\frac{1}{4}\left(\tilde{V}_{--}(R)-2 \tilde{V}_{+-}(R)+\tilde{V}_{++}(R)\right), \\
& J_{x}(R)=2\left(\tilde{W}_{+-}(R)+\tilde{W}_{++}(R)\right) \\
& J_{y}(R)=2\left(\tilde{W}_{+-}(R)-\tilde{W}_{++}(R)\right)
\end{aligned}
$$

$$
J_{\|}(R)=\frac{1}{4}\left(\tilde{V}_{--}(R)-\tilde{V}_{++}(R)\right),
$$

where the functions $\tilde{W}_{\alpha \beta}$ and $\tilde{V}_{\alpha \beta}$ are effective radial dependent steplike potentials,

$$
\begin{aligned}
\tilde{V}_{\alpha \alpha}(R)= & \frac{\Omega_{\bar{\sigma}}^{2}}{2 \Delta_{\bar{\sigma}}}-\frac{\Omega_{\bar{\sigma}}^{4}}{4 \Delta_{\bar{\sigma}}^{3}} \\
& +\frac{\Omega_{\bar{\alpha}}^{4}}{4 \Delta_{\bar{\alpha}}^{2}} \frac{V_{++}-2 \Delta_{\alpha}}{W_{++}^{2}-\left(V_{++}-2 \Delta_{+}\right)\left(V_{++}-2 \Delta_{-}\right)}, \\
\tilde{V}_{+-}(R)= & \frac{\Omega_{-}^{2}}{4 \Delta_{-}}+\frac{\Omega_{+}^{2}}{4 \Delta_{+}}-\frac{\Omega_{+}^{2} \Omega_{-}^{2}}{16 \Delta_{+}^{2} \Delta_{-}} \\
& -\frac{\Omega_{+}^{2} \Omega_{-}^{2}}{16 \Delta_{-}^{2} \Delta_{+}}-\frac{\Omega_{-}^{4}}{16 \Delta_{-}^{3}}-\frac{\Omega_{+}^{4}}{16 \Delta_{+}^{3}} \\
& +\frac{\Delta_{ \pm}^{2} \Omega_{+}^{2} \Omega_{-}^{2}}{16 \Delta_{+}^{2} \Delta_{-}^{2}} \frac{\left(\Delta_{ \pm}-V_{+-}\right)}{\left(\Delta_{ \pm}-V_{+-}\right)^{2}-W_{+-}^{2}}, \\
\tilde{W}_{+-}(R) & \left.=\frac{\Omega_{+}^{2} \Omega_{-}^{2}}{16 \Delta_{+}^{2} \Delta_{-}^{2}} \frac{\Delta_{ \pm}^{2} W_{+-}}{\left(\Delta_{ \pm}-V_{+-}\right)^{2}-W_{+-}^{2}}, \quad \text { (A7 }\right)
\end{aligned}
$$

$\tilde{W}_{++}(R)=\frac{\Omega_{+}^{2} \Omega_{-}^{2}}{4 \Delta_{+} \Delta_{-}} \frac{W_{++}}{W_{++}^{2}-\left(V_{++}-2 \Delta_{+}\right)\left(V_{++}-2 \Delta_{-}\right)}$,

written in terms of the $n^{2} P_{1 / 2}$ van der Waals potentials $V_{\alpha \beta}$ and $W_{\alpha \beta}$. Note that the single-particle light shifts have been included in the above expressions. Moreover, $\tilde{V}_{+-}=\tilde{V}_{-+}$ and we have defined $\Delta_{ \pm}=\Delta_{+}+\Delta_{-}$and $\bar{\alpha}=-\alpha$. In the parameter region $\Delta_{+-}<0, \Delta_{+} / \Delta_{-}<0$ resonant Rydberg excitations are avoided for all values of $R$. For atomic orientation $\theta=\pi / 2$ (polar), $\phi=0$ (azimuthal) the van der Waals potentials are

$$
V_{\alpha \beta}=\frac{c_{\alpha \beta}}{R^{6}}, \quad W_{+-}=\frac{w}{R^{6}}=-\frac{1}{3} W_{++},
$$

where the so-called $C_{6}$ coefficients $c_{\alpha \beta}$ and $w$ are obtained from second-order perturbation theory and are given by

$$
\begin{array}{r}
c_{++}=\frac{2}{81}\left(5 C_{6}^{(a)}+14 C_{6}^{(b)}+8 C_{6}^{(c)}\right), \\
c_{+-}=\frac{2}{81}\left(C_{6}^{(a)}+10 C_{6}^{(b)}+16 C_{6}^{(c)}\right), \\
w=\frac{2}{81}\left(C_{6}^{(a)}+C_{6}^{(b)}-2 C_{6}^{(c)}\right) .
\end{array}
$$

The individual channel coefficients $C_{6}^{(v)}, v=a, b, c$ are independent of magnetic quantum numbers and charac- 


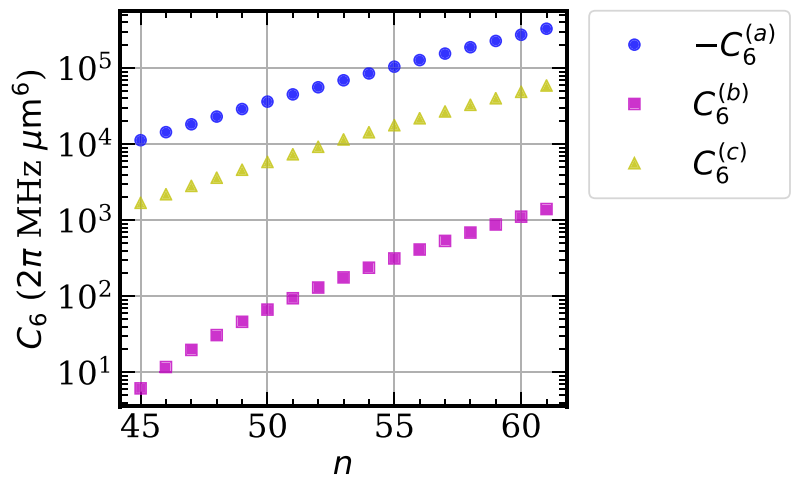

FIG. 9. The $C_{6}^{(v)}$ coefficients as a function of the principal quantum number for the $n^{2} P_{1 / 2}$ manifold.

terize the interaction strength. There is one channel for each nonvanishing matrix element of the dipole-dipole interaction potential [42],

$$
\begin{aligned}
& a: P_{1 / 2}+P_{1 / 2} \rightarrow S_{1 / 2}+S_{1 / 2}, \\
& b: P_{1 / 2}+P_{1 / 2} \rightarrow D_{3 / 2}+D_{3 / 2}, \\
& c: P_{1 / 2}+P_{1 / 2} \rightarrow D_{3 / 2}+S_{1 / 2},
\end{aligned}
$$

and each $C_{6}^{(v)}$ is calculated from the radial part of the dipole-dipole matrix element [87],

$$
C_{6}^{(v)}=\sum_{n_{\alpha} n_{\beta}} \frac{e^{4}}{\delta_{\alpha \beta}}\left(R_{n l}^{n_{\alpha} l_{\alpha}} R_{n l}^{n_{\beta} l_{\beta}}\right)^{2}
$$

where

$$
R_{n l}^{n_{i} l_{i}}=\int d r r^{2} \psi_{n, l, j}(r)^{*} r \psi_{n_{i}, l_{i}, j_{i}}(r)
$$

and $\delta_{\alpha \beta}$ is the energy defect between levels $n_{\alpha}$ and $n_{\beta}$.

To numerically obtain the coefficients given in Eq. (A15) and, consequently, the steplike potentials given in Eqs. (A5) and (A7), we use the ARC PYTHON library for alkali Rydberg atoms [88]. Numerical calculation results are shown in Fig. 9 as a function of the principal quantum number for the $n^{2} P_{1 / 2}$ manifold. For $n=60$, as used in the main text, we find

$$
\begin{aligned}
& -C_{6}^{(a)} \approx 2 \pi \times 2.7 \times 10^{5} \mathrm{MHz} \mu \mathrm{m}^{6}, \\
& C_{6}^{(b)} \approx 2 \pi \times 1.1 \times 10^{3} \mathrm{MHz} \mu \mathrm{m}^{6}, \\
& C_{6}^{(c)} \approx 2 \pi \times 4.9 \times 10^{4} \mathrm{MHz} \mu \mathrm{m}^{6} .
\end{aligned}
$$

\section{APPENDIX B: MAGNETIC FIELD $\boldsymbol{J}_{\|}$}

Besides the $J_{\alpha}(R)$ coefficients, the Rydberg dressing generates an effective magnetic field term $J_{\|}\left(Z_{a}+Z_{b}\right)$ in the interaction energy. Under the influence of this term, the Bell states of the $a b$ pair are no longer eigenstates of the interaction. To obtain the spin-dependent potential landscapes given by the eigenvalues in Eqs. (5)-(8), we need to cancel $J_{\|}$by applying an external spatially dependent static field. How large does such a field need to be? A plot of $J_{\|}$can be seen in Fig. 10.

Note that $\left\langle J_{\|}\right\rangle \approx 1401 \mathrm{kHz}$. Considering the Landé factor $\left|g_{F}\right| \approx 0.70 \mathrm{MHz} / \mathrm{G}$ for the $5^{2} S_{1 / 2}$ states [89], this effective magnetic field can be canceled by an additional weak nonhomogeneous field of order of magnitude $\left|B_{c}\right| \approx$ $2 \mathrm{G}$.

\section{APPENDIX C: ALTERNATIVE SPIN PATTERN}

Alternative spin-dependent potentials, defined by parameters different from the ones employed in the main text, are shown in Fig. 11. Here, we consider detunings $\Delta_{+}=-2 \pi \times 70 \mathrm{MHz}$ and $\Delta_{-}=2 \pi \times 30 \mathrm{MHz}$, Rabi frequencies $\Omega_{+}=\Omega_{-}=-2 \pi \times 7 \mathrm{MHz}$, and the trap potential

$$
V_{t}(R)=V_{0}(R-\delta)^{2}
$$

where $V_{0}=15 \mathrm{kHz} / \mu \mathrm{m}^{2}$ and $\delta=2.30 \mu \mathrm{m}$. Note that the resulting landscapes in Fig. 11(c) suggest $\left|\phi^{-}\right\rangle$as a protected state, while the corrective loops $L 1$ and $L 2$ should be reversed with respect to the choice discussed in the main text. The effective magnetic field has a mean value $\left\langle J_{\|}\right\rangle \approx 1803 \mathrm{kHz}$, which requires a slightly higher compensating magnetic field but still on the order of a few gauss. The spatial profile $J_{\|}(R)$ is shown in Fig. 12.

\section{APPENDIX D: OPTIMAL OPERATION}

To evaluate the effect of positioning of the correctors $L 1$ and $L 2$, we run SMMC, as described in the main text, for different values of the positions $R_{L 1}=-R_{L 2}$.

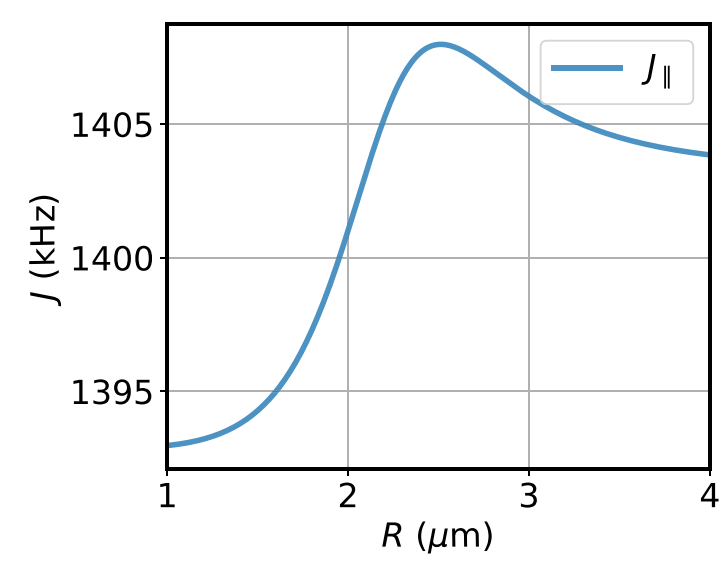

FIG. 10. The $J_{\|}$profile. 
(a)

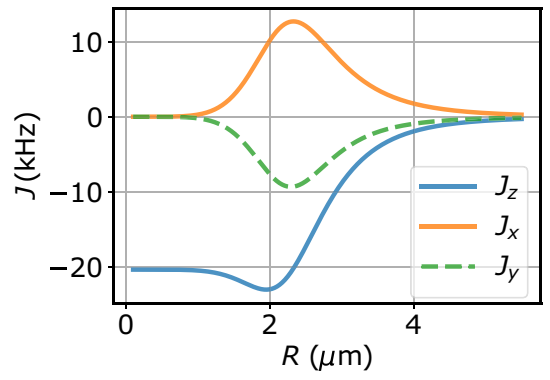

(b)

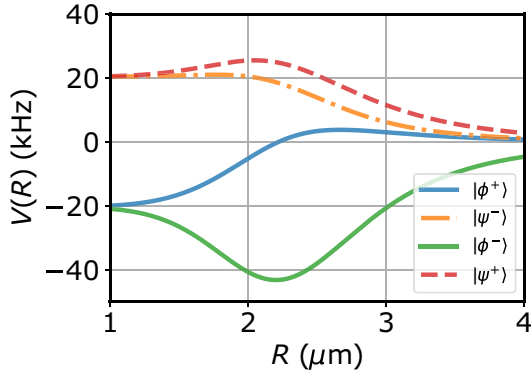

(c)

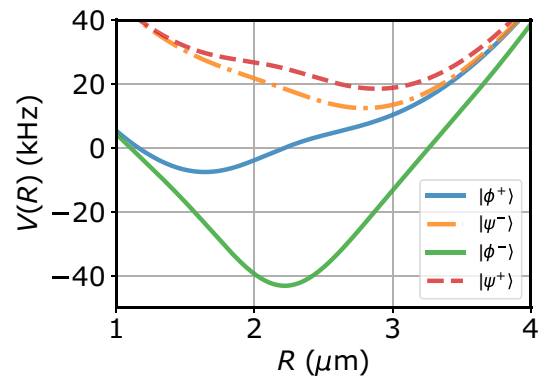

FIG. 11. (a) The alternative spin-pattern profile. (b) The resulting spin-dependent potentials. (c) The resulting spin-dependent potentials after adding the spin-independent harmonic potential.

Figure 13 shows traces of the overlap $F$ as a function of time. Each trace corresponds to a different corrector position (see caption) and the overlap of free spins under the action of the depolarizing channel is shown as the gray dashed line for comparison. The points in Fig. 8 (see the main text) are obtained by time averaging the overlap above $10 \mathrm{~ms}$ for each of the traces in Fig. 13.

We can see that if the positions of the correctors are too close to the equilibrium position of the atom, the overlap quickly decays due to internal errors, which occur when a quantum fluctuation in the atomic position places it near the corrective site. This fast drop in overlap can be mitigated by positioning the correctors further apart from the $\left|\phi^{+}\right\rangle$equilibrium point. There is, however, a trade-off: the maximum steady-state overlap of approximately $70 \%$ is reached for a position $\left|R_{L 1}\right| \approx 0.63 \mu \mathrm{m}$, while placing the correctors further apart than that reduces the correction rates below the decoherence rate and consequently the steady-state overlap.

Decoherence causes the overlap to decrease exponentially, according to $e^{-\Gamma t}=e^{-t / \tau_{D}}$, where $\tau_{D}=\Gamma^{-1}=$ $(100 \mathrm{~Hz})^{-1}=10 \mathrm{~ms}$ is the characteristic decay time of the

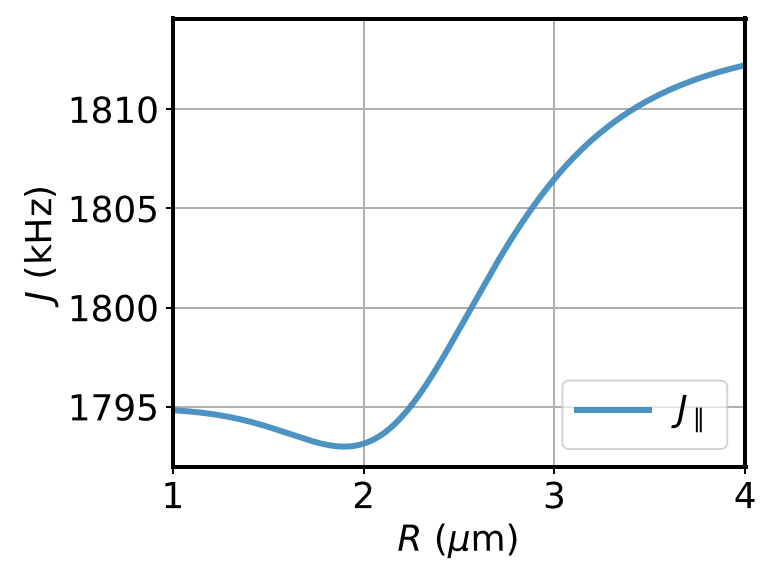

FIG. 12. The alternative effective magnetic field. system. Decoherence effectively freezes when the system reaches its steady state, which happens after a stabilization time $t_{s}$ elapses. From Fig. 6 in the main text, we see that $t_{s} \approx 4 \mathrm{~ms}$, yielding an expected overlap of $F \approx e^{-t_{s} / \tau_{D}} \approx$ 0.67 , in accordance with the simulation results.
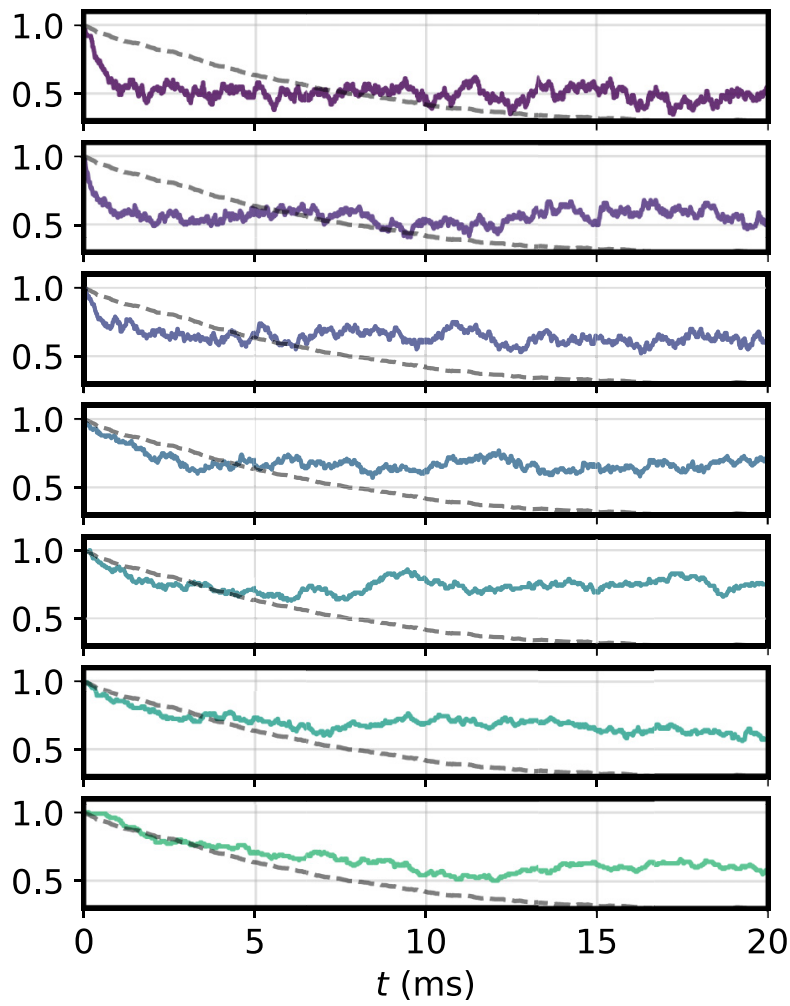

FIG. 13. The overlap $F$ for different values of the corrector position $\left|R_{L 1}\right|=\left|R_{L 2}\right|$ obtained from simulating $10^{2}$ quantum trajectories. For each trace position, the values are $(0.47,0.52,0.58,0.63,0.69,0.74,0.80) \mu \mathrm{m}$ from top to bottom, respectively. The gray dashed lines represent the overlap of free decohering spins, for comparison. All remaining parameters are the same as in Fig. 6 in the main text. 
[1] C. H. Bennet, The thermodynamics of computation-A review, Int. J. Theor. Phys. 21, 905 (1982).

[2] D. S. Goodsell, The Machinery of Life (Copernicus, New York, 2009).

[3] B. Alberts, D. Bray, K. Hopkin, A. D. Johnson, J. Lewis, M. Raff, K. Roberts, and P. Walter, Essential Cell Biology (Garland Science, Philadelphia, PA, 2009), 3rd ed.

[4] R. Milo and R. Phillips, Cell Biology by the Numbers (Garland Science, New York, 2015).

[5] R. P. Feynman, There's plenty of room at the bottom, Eng. Sci. 23, 22 (1960).

[6] L. Zhang, V. Marcos, and D. A. Leigh, Molecular machines with bio-inspired mechanisms, PNAS 115, 9397 (2018).

[7] V. Serreli, C. F. Lee, E. R. Kay, and D. A. Leigh, A molecular information ratchet, Nature 445, 523 (2007).

[8] C. Cheng, P. R. McGonigal, S. T. Schneebeli, H. Li, N. A. Vermeulen, C. Ke, and J. F. Stoddart, An artificial molecular pump, Nat. Nanotechnol. 10, 547 (2015).

[9] S. Kassem, T. van Leeuwen, A. S. Lubbe, M. R. Wilson, and B. L. Feringa, Artificial molecular motors, Chem. Soc. Rev. 46, 2592 (2017).

[10] J. A. Doudna and S. H. Sternberg, A Crack in Creation: Gene Editing and the Unthinkable Power to Control Evolution (Houghton Mifflin Harcourt, Boston, 2017).

[11] W. Bialek, Biophysics: Searching for Principles (Princeton University Press, Oxford, 2012).

[12] A. Chakraborty, C. A. Meng, and S. M. Block, Observing single RNA polymerase molecules down to base-pair resolution, Opt. Tweezers (Humana Press, New York, NY, 2017), Vol. 1486.

[13] P. C. Bressloff, Stochastic Processes in Cell Biology (Springer International Publishing, Switzerland, 2014).

[14] Google AI Quantum, Hartree-Fock on a superconducting qubit quantum computer, Science 369, 1084 (2020).

[15] C. Outeiral, M. Strahm, J. Shi, G. M. Morris, S. C. Benjamin, and C. M. Deane, The prospects of quantum computing in computational molecular biology, WIREs Comput. Mol. Sci. 11, e1481 (2020).

[16] P. S. Emani, J. Warrell, A. Anticevic, S. Bekiranov, M. Gandal, M. J. McConnell, G. Sapiro, A. Aspuru-Guzik, J. T. Baker, M. Bastiani, and J. D. Murray, Quantum computing at the frontiers of biological sciences, Nat. Methods 18, 701 (2021).

[17] H. Frauenfelder, Ask not what physics can do for biology — ask what biology can do for physics, Phys. Biol. 11, 053004 (2014).

[18] T. Guerreiro, Quantum molecular robots (Quantum Sci. Technol. 6, 025006 (2020).

[19] L. D. Carr, D. DeMille, R. V. Krems, and J. Ye, Cold and ultracold molecules: Science, technology and applications, New J. Phys. 11, 055049 (2009).

[20] R. V. Krems, Molecules in Electromagnetic Fields: From Ultracold Physics to Controlled Chemistry (Wiley, New York, 2018).

[21] B. Lau, O. Kedem, J. Schwabacher, D. Kwasnieski, and E. A. Weiss, An introduction to ratchets in chemistry and biology, Mater. Horiz. 4, 310 (2017).

[22] D. Budker, D. Kimball, and D. DeMille, Atomic Physics: An Exploration Through Problems and Solutions (Oxford University Press, New York, 2014).
[23] S. Ospelkaus, K. K. Ni, D. Wang, M. H. De Miranda, B. Neyenhuis, G. Quéméner, P. S. Julienne, J. L. Bohn, D. S. Jin, and J. Ye, Quantum-state controlled chemical reactions of ultracold potassium-rubidium molecules, Science 327, 853 (2010).

[24] L. R. Liu, J. T. Zhang, Y. Yu, N. R. Hutzler, Y. Liu, T. Rosenband, and K. K. Ni, Ultracold molecular assembly, ArXiv:1701.03121.

[25] M. B. Plenio, S. F. Huelga, A. Beige, and P. L. Knight, Cavity loss induced generation of entangled atoms, Phys. Rev. A 59, 2468 (1999).

[26] M. B. Plenio and S. F. Huelga, Entangled Light from White Noise, Phys. Rev. Lett. 88, 197901 (2002).

[27] S. Diehl, A. Micheli, A. Kantian, B. Kraus, H. P. Büchler, and P. Zoller, Quantum states and phases in driven open quantum systems with cold atoms, Nat. Phys. 4, 878 (2008).

[28] F. Verstraete, M. M. Wolf, and J. I. Cirac, Quantum computation, quantum state engineering, and quantum phase transitions driven by dissipation, Nat. Phys. 5, 633 (2009).

[29] G. Vacanti and A. Beige, Cooling atoms into entangled states, New J. Phys. 11, 083008 (2009).

[30] F. Reiter, M. J. Kastoryano, and A. S. Sorensen, Driving two atoms in an optical cavity into an entangled steady state using engineered decay, New J. Phys. 14, 053022 (2012).

[31] J. B. Brask, N. Brunner, G. Haack, and M. Huber, Autonomous quantum thermal machine for generating steady-state entanglement, New J. Phys. 17, 113029 (2015).

[32] F. Reiter, A. S. Sorensen, P. Zoller, and C. A. Muschik, Dissipative quantum error correction and application to quantum sensing with trapped ions, Nat. Commun. 8, 1822 (2017).

[33] C. Cheng, P. R. McGonigal, J. F. Stoddart, and R. D. Astumian, Design and synthesis of nonequilibrium systems, ACS Nano 9, 8672 (2015).

[34] A. Y. Kitaev, Unpaired Majorana fermions in quantum wires, Phys.-Usp. 44, 131 (2001).

[35] A. G. Fowler, M. Mariantoni, J. M. Martinis, and A. N. Cleland, Surface codes: Towards practical large-scale quantum computation, Phys. Rev. A 86, 032324 (2012).

[36] Q. Wei, S. Kais, B. Friedrich, and D. Herschbach, Entanglement of polar molecules in pendular states, J. Chem. Phys. 134, 124107 (2011).

[37] J. Pietraszewicz, T. Sowinski, M. Brewczyk, M. Lewenstein, and M. Gajda, Spin dynamics of two bosons in an optical lattice site: A role of anharmonicity and anisotropy of the trapping potential, Phys. Rev. A 88, 013608 (2013).

[38] S. Choi, J. Choi, R. Landig, G. Kucsko, H. Zhou, J. Isoya, F. Jelezko, S. Onoda, H. Sumiya, V. Khemani, and C. Von Keyserlingk, Observation of discrete time-crystalline order in a disordered dipolar many-body system, Nature 543, 221 (2017).

[39] S. Weinberg, Lectures on Quantum Mechanics (Cambridge University Press, New York, 2012).

[40] D. Porras and J. I. Cirac, Effective Quantum Spin Systems with Ion Traps, Phys. Rev. Lett. 92, 207901 (2004).

[41] K. Kim, M. S. Chang, S. Korenblit, R. Islam, E. E. Edwards, J. K. Freericks, G. D. Lin, L. M. Duan, and C. Monroe, Quantum simulation of frustrated Ising spins with trapped ions, Nature 465, 590 (2010). 
[42] A. W. Glaetzle, M. Dalmonte, R. Nath, C. Gross, I. Bloch, and P. Zoller, Designing Frustrated Quantum Magnets with Laser-Dressed Rydberg Atoms, Phys. Rev. Lett. 114, 173002 (2015).

[43] R. M. W. van Bijnen and T. Pohl, Quantum Magnetism and Topological Ordering via Enhanced Rydberg-Dressing Near Forster-Resonances, Phys. Rev. Lett. 114, 243002 (2015).

[44] A. Micheli, G. K. Brennen, and P. Zoller, A toolbox for lattice spin models with polar molecules, Nat. Phys. 2, 341 (2006).

[45] G. K. Brennen, A. Micheli, and P. Zoller, Designing spin-1 lattice models using polar molecules, New J. Phys. 9, 138 (2007).

[46] D. Gottesman, A class of quantum error-correcting codes saturating the quantum hamming bound, Phys. Rev. A 54, 1862 (1996).

[47] R. Laflamme, C. Miquel, J. P. Paz, and W. H. Zurek, Perfect Quantum Error Correcting Code, Phys. Rev. Lett. 77, 198 (1996).

[48] C. H. Bennett, Notes on Landauer's principle, reversible computation and Maxwell's Demon, Stud. Hist. Philos. Mod. Phys. 34, 501 (2003).

[49] J. Bub, Maxwell's demon and the thermodynamics of computation, Stud. Hist. Philos. Mod. Phys. 32, 569 (2001).

[50] J. Gea-Banacloche, Minimum Energy Requirements for Quantum Computation, Phys. Rev. Lett. 89, 217901 (2002).

[51] D. J. Bedingham and O. J. E. Maroney, The thermodynamic cost of quantum operations, New J. Phys. 18, 113050 (2016).

[52] S. Deffner, Energetic cost of Hamiltonian quantum gates, ArXiv:2102.05118 (2021).

[53] J. P. Peterson, R. S. Sarthour, A. M. Souza, I. S. Oliveira, J. Goold, K. Modi, D. O. Soares-Pinto, and L. C. Céleri, Experimental demonstration of information to energy conversion in a quantum system at the Landauer limit, Proc. R. Soc. A 472, 20150813 (2016).

[54] R. Gaudenzi, E. Burzurí, S. Maegawa, H. S. Van Der Zant, and F. Luis, Quantum Landauer erasure with a molecular nanomagnet, Nat. Phys. 14, 565 (2018).

[55] V. Cimini, S. Gherardini, M. Barbieri, I. Gianani, M. Sbroscia, L. Buffoni, M. Paternostro, and F. Caruso, Experimental characterization of the energetics of quantum logic gates, npj Quantum Inf. 6, 96 (2020).

[56] Y. Y. Jau, A. M. Hankin, T. Keating, I. H. Deutsch, and G. W. Biedermann, Entangling atomic spins with a Rydbergdressed spin-flip blockade, Nat. Phys. 12, 71 (2016).

[57] Jun Ye, H. J. Kimble, and H. Katori, Quantum state engineering and precision metrology using state-insensitive light traps, Science 320, 1734 (2008).

[58] S. Zhang, F. Robicheaux, and M. Saffman, Magicwavelength optical traps for Rydberg atoms, Phys. Rev. A 84, 043408 (2011).

[59] A. G. Boetes, R. V. Skannrup, J. B. Naber, S. J. J. M. F. Kokkelmans, and R. J. C. Spreeuw, Trapping of Rydberg atoms in tight magnetic microtraps, Phys. Rev. A 97, 013430 (2018).

[60] V. Paulisch, H. Rui, H. K. Ng, and B. G. Englert, Beyond adiabatic elimination: A hierarchy of approximations for multi-photon processes, EPJ-Plus 129, 12 (2014).
[61] A. W. Glaetzle, M. Dalmonte, R. Nath, I. Rousochatzakis, R. Moessner, and P. Zoller, Quantum Spin Ice and Dimer Models with Rydberg Atoms, Phys. Rev. X 4, 041037 (2014).

[62] D. Kielpinski, D. Kafri, M. J. Woolley, G. J. Milburn, and J. M. Taylor, Quantum Interface between an Electrical Circuit and a Single Atom, Phys. Rev. Lett. 108, 130504 (2012).

[63] A. André, D. DeMille, J. M. Doyle, M. D. Lukin, S. E. Maxwell, P. Rabl, R. J. Schoelkopf, and P. Zoller, A coherent all-electrical interface between polar molecules and mesoscopic superconducting resonators, Nat. Phys. 2, 636 (2006).

[64] L. Martinetz, K. Hornberger, J. Millen, M. S. Kim, and B. A. Stickler, Quantum electromechanics with levitated nanoparticles, npj Quantum Inf. 6, 101 (2020).

[65] Y. Kubo, C. Grezes, A. Dewes, T. Umeda, J. Isoya, H. Sumiya, N. Morishita, H. Abe, S. Onoda, T. Ohshima, and V. Jacques, Hybrid Quantum Circuit with a Superconducting Qubit Coupled to a Spin Ensemble, Phys. Rev. Lett. 107, 220501 (2011).

[66] D. Jaksch, H. J. Briegel, J. I. Cirac, C. W. Gardiner, and P. Zoller, Entanglement of Atoms via Cold Controlled Collisions, Phys. Rev. Lett. 82, 1975 (1999).

[67] R. P. Feynman, Feynman Lectures on Physics (AddisonWesley, Redwood City, 2006), Vol. III, Chapter 9.

[68] R. Wynands and S. Weyers, Atomic fountain clocks, Metrologia 42, S64 (2005).

[69] D. M. Weld, P. Medley, H. Miyake, D. Hucul, D. E. Pritchard, and W. Ketterle, Spin Gradient Thermometry for Ultracold Atoms in Optical Lattices, Phys. Rev. Lett. 103, 245301 (2009).

[70] A. J. Daley, P. O. Fedichev, and P. Zoller, Single-atom cooling by superfluid immersion: A nondestructive method for qubits, Phys. Rev. A 69, 022306 (2004).

[71] A. Griessner, D. Jaksch, and P. Zoller, Cavity-assisted nondestructive laser cooling of atomic qubits, J. Phys. B: At. Mol. Opt. Phys. 37, 1419 (2004).

[72] R. Belyansky, J. T. Young, P. Bienias, Z. Eldredge, A. M. Kaufman, P. Zoller, and A. V. Gorshkov, Nondestructive Cooling of an Atomic Quantum Register via StateInsensitive Rydberg Interactions, Phys. Rev. Lett. 123, 213603 (2019).

[73] I. I. Beterov, I. I. Ryabtsev, D. B. Tretyakov, and V. M. Entin, Quasiclassical calculations of blackbody-radiationinduced depopulation rates and effective lifetimes of Rydberg $n S, n P$ and $n D$ alkali-metal atoms with $n \leq 80$, Phys. Rev. A 79, 052504 (2009).

[74] J. Preskill, Quantum information lecture notes, Chapter 7, http://theory.caltech.edu/ preskill/ph229/notes/chap7.pdf.

[75] C. Misner, K. Thorne, and W. Zurek, John Wheeler, relativity, and quantum information, Phys. Today 62, 40 (2009).

[76] N. V. Morrow, S. K. Dutta, and G. Raithel, Feedback Control of Atomic Motion in an Optical Lattice, Phys. Rev. Lett. 88, 093003 (2002).

[77] P. Bushev, D. Rotter, A. Wilson, F. Dubin, C. Becher, J. Eschner, R. Blatt, V. Steixner, P. Rabl, and P. Zoller, Feedback Cooling of a Single Trapped Ion, Phys. Rev. Lett. 96, 043003 (2006). 
[78] L. Magrini, P. Rosenzweig, C. Bach, A. DeutschmannOlek, S. G. Hofer, S. Hong, N. Kiesel, A. Kugi, and M. Aspelmeyer, Real-time optimal quantum control of mechanical motion at room temperature, Nature 595, 373 (2021).

[79] J. McKeever, J. R. Buck, A. D. Boozer, A. Kuzmich, H. C. Nägerl, D. M. Stamper-Kurn, and H. J. Kimble, StateInsensitive Cooling and Trapping of Single Atoms in an Optical Cavity, Phys. Rev. Lett. 90, 133602 (2003).

[80] J. R. Johansson, P. D. Nation, and F. Nori, QuTiP 2: A PYTHON framework for the dynamics of open quantum systems, Comput. Phys. Commun. 184, 1234 (2013).

[81] F. Wilczek, Quantum Time Crystals, Phys. Rev. Lett. 109, 160401 (2012).

[82] J. Zhang, P. W. Hess, A. Kyprianidis, P. Becker, A. Lee, J. Smith, G. Pagano, I. D. Potirniche, A. C. Potter, A. Vishwanath, and N. Y. Yao, Observation of a discrete time crystal, Nature 543, 217 (2017).

[83] G. J. Milburn and H. M. Wiseman, Quantum Measurement and Control (Cambridge University Press, New York, 2009).
[84] C. Ahn, A. C. Doherty, and A. J. Landahl, Continuous quantum error correction via quantum feedback control, Phys. Rev. A 65, 042301 (2002).

[85] T. Vicsek, A. Czirók, E. Ben-Jacob, I. Cohen, and Shochet O. Novel, Novel Type of Phase Transition in a System of Self-Driven Particles, Phys. Rev. Lett. 75, 1226 (1995).

[86] D. Geyer, A. Morin, and D. Bartolo, Sounds and hydrodynamics of polar active fluids, Nat. Mater. 17, 789 (2018).

[87] T. G. Walker and M. Saffman, Consequences of Zeeman degeneracy for van der Waals blockade between Rydberg atoms, Phys. Rev. A 77, 032723 (2008).

[88] N. Šibalic, J. D. Pritchard, C. S. Adams, and K. J. Weatherill, ARC: An open source library for calculating properties of alkali Rydberg atoms, Comput. Phys. Commun. 220, 319 (2017).

[89] S. Bize, Y. Sortais, M. S. Santos, C. Mandache, A. Clairon, and C. Salomon, High-accuracy measurement of the ${ }^{8} 7 \mathrm{Rb}$ ground-state hyperfine splitting in an atomic fountain, Europhys. Lett. 45, 558 (1999). 